\title{
Numerical study of Hele-Shaw flow with suction
}

\author{
Hector D. Ceniceros, Thomas Y. Hou, and Helen Si \\ Applied Mathematics, California Institute of Technology, Pasadena, California 91125
}

(Received 30 July 1998; accepted 27 May 1999)

\begin{abstract}
We investigate numerically the effects of surface tension on the evolution of an initially circular blob of viscous fluid in a Hele-Shaw cell. The blob is surrounded by less viscous fluid and is drawn into an eccentric point sink. In the absence of surface tension, these flows are known to form cusp singularities in finite time. Our study focuses on identifying how these cusped flows are regularized by the presence of small surface tension, and what the limiting form of the regularization is as surface tension tends to zero. The two-phase Hele-Shaw flow, known as the Muskat problem, is considered. We find that, for nonzero surface tension, the motion continues beyond the zero-surface-tension cusp time, and generically breaks down only when the interface touches the sink. When the viscosity of the surrounding fluid is small or negligible, the interface develops a finger that bulges and later evolves into a wedge as it approaches the sink. A neck is formed at the top of the finger. Our computations reveal an asymptotic shape of the wedge in the limit as surface tension tends to zero. Moreover, we find evidence that, for a fixed time past the zero-surface-tension cusp time, the vanishing surface tension solution is singular at the finger neck. The zero-surface-tension cusp splits into two corner singularities in the limiting solution. Larger viscosity in the exterior fluid prevents the formation of the neck and leads to the development of thinner fingers. It is observed that the asymptotic wedge angle of the fingers decreases as the viscosity ratio is reduced, apparently towards the zero angle (cusp) of the zero-viscosity-ratio solution. (C) 1999 American Institute of Physics. [S1070-6631(99)03909-4]
\end{abstract}

\section{INTRODUCTION}

A Hele-Shaw cell is a device for investigating the twodimensional flow of viscous fluids in a narrow gap between two parallel plates. Through the similarity in their governing equations, Hele-Shaw flows are linked to saturated flows in porous media. One of the main sources of interest in this type of flows is the oil industry. In particular, Hele-Shaw flows with suction are relevant to the process of oil recovery. In these flows, a blob of viscous fluid, surrounded by inviscid or less viscous fluid, is drawn radially into a point sink. The more viscous fluid can be associated with oil, surrounded by water (inviscid fluid), that is recovered through a well (sink).

Laboratory experiments ${ }^{1}$ show that Hele-Shaw flows with suction can develop long "fingers" of the less viscous fluid that encroach upon the more viscous fluid. These fingers reach the sink before all the more viscous fluid is sucked out. In the oil analogy, this fingering process could reduce the amount of recoverable oil. From physical grounds, it is believed that surface tension plays a crucial role in the development and width selection of the fingers. In the absence of surface tension, fingering is not observed. Instead, solutions of the Hele-Shaw equations, for the suction flow with inviscid surrounding fluid, are known to form finite-time singularities before the fluid interface reaches the sink. These singularities are generally in the form of $\frac{3}{2}$-power cusps. ${ }^{2,3}$

From the analytical point of view, very little is known about the Hele-Shaw solutions in the presence of surface tension. There is a local existence result by Duchon and Robert, ${ }^{4}$ and a global in time result for initial data close to a circle by Constantin and Pugh. ${ }^{5}$ Tian $^{6}$ shows that singularity formation is inevitable if the center of the viscous blob is not at the sink. However, the type of singularity is still unknown. The singularity could be caused by the interface reaching the sink or by other means.

It is natural to use the knowledge of the zero-surfacetension solutions to study the asymptotic effects of surface tension as a perturbation parameter. However, a perturbation analysis is difficult due to the ill posedness of the underlying zero-surface-tension problem ${ }^{7,8}$ and to the singular nature of the perturbation. Instead, Howison et al. ${ }^{2}$ propose an asymptotic model in which small surface tension would cause the interface in the neighborhood of the cusp to propagate rapidly as a narrow jet, analogous to a thin crack. However, this so-called "crack" model relies on the notion of a self-similar steady-state solution whose existence is unknown. Thus, the effects of very small surface tension past the cusp time remain unclear.

Here, we investigate numerically how surface tension regularizes the cusped flows and what the limiting form of this regularization is as surface tension tends to zero. We consider the two-phase Hele-Shaw flow, known as the Muskat problem, ${ }^{9}$ and study also the effects that the viscosity of the surrounding fluid has on the Hele-Shaw sink flow. We employ a highly accurate and efficient boundary integral method developed by Hou et al. ${ }^{10}$ This method allows us to follow accurately the interface motion after the zero-surfacetension cusp time $t_{c}$, for very small values of surface tension and several viscosity ratios. With high resolution, our computations proceed up to very close to the moment when the 
interface touches the sink and the solution breaks down in the classical sense.

Kelly and Hinch ${ }^{11}$ study numerically the effects of surface tension on the Hele-Shaw flow with suction when the surrounding fluid is inviscid. Their computations show that surface tension indeed regularizes the cusped flow and that a smooth narrow finger develops past $t_{c}$. However, as we show here, Kelly and Hinch computations lack the high accuracy necessary to capture the interface behavior for sufficiently small surface tension. In addition, for a given surface tension, high resolution is needed to compute the fast interface motion as the finger gets very close to the sink. In fact, recent computations by Nie and Tian ${ }^{12}$ provide strong evidence that the flow develops a curvature singularity (in the form of a corner) when the interface reaches the sink. However, Nie and Tian do not address the limiting behavior of the interface as surface tension tends to zero.

Numerical computations of unstable Hele-Shaw flows are known to be difficult due to the ill posedness of the zero-surface-tension problem. ${ }^{13,14}$ For sufficiently small surface tension, even perturbations at the round-off error level can lead to a rapid growth of the solution high-frequency components. ${ }^{15,16}$ In addition, surface tension introduces highorder derivative terms that couple with the interface dynamics in a nonlinear and nonlocal manner. These terms lead to a severe time-step stability constraint or stiffness for explicit time-integration schemes. In their method, Hou et al. ${ }^{10}$ effectively identify and separate the terms causing the stiffness in a form that makes implicit discretizations easy to implement. Here, we apply this method to a spectrally accurate discretization in space combined with a fourth-order in time implicit/explicit multi-step scheme.

Our computations focus on the evolution of an initially circular blob of viscous fluid surrounded by less viscous fluid. The blob is drawn into a point sink located inside it but not at its initial center. We find that, for nonzero surface tension, the flow continues beyond the zero-surface-tension cusp time, and breaks down only when the interface touches the sink. When the viscosity of the surrounding fluid is small or negligible, the interface develops a finger that later evolves into a wedge as it approaches the sink. As in the cases reported by Nie and Tian, ${ }^{12}$ our computations strongly suggest that the Hele-Shaw solutions, for this type of geometry, generically break down by developing a corner at the tip of the wedge when the interface reaches the sink.

As surface tension is decreased, our numerical results show several new interesting phenomena. An asymptotic shape of the fingers is observed at the late stage of the interface motion and a wedge angle at the tip of the finger is selected in the limit as surface tension tends to zero. Moreover, for sufficiently small values of surface tension a welldefined neck develops at the top of the finger near $t_{c}$. The developing finger bulges but, being drawn strongly by the sink, quickly evolves into the wedge. The bulging of the finger contradicts the "crack" model of Howison et al. It is conceivable that the neck and the bulging of the finger are due to the influence of the zero-surface-tension singularity. In fact, our computations suggest that the vanishing surface tension solution is singular at the finger neck, for any fixed time past $t_{c}$ but not greater than the asymptotic time at which the interface reaches the sink. The continuation solution selected by the limit apparently splits the zero-surface-tension cusp into two corner singularities at the finger neck.

Even in the absence of surface tension, very little is known about the corresponding Muskat problem, i.e., when the viscosity of the surrounding fluid is taken into consideration. We find here that the viscosity of the exterior fluid alone does not prevent the formation of cusps. It only delays them. In the absence of surface tension, we observe generic $\frac{3}{2}$-power cusps just as in the case of inviscid exterior fluid. However, as the viscosity ratio is decreased, we find that the zero-surface-tension cusps develop closer to the sink. In the presence of surface tension, the interface behavior for small viscosity ratios differs significantly from that corresponding the case of inviscid exterior fluid. We observe that large viscosity in the exterior fluid prevents the formation of the neck and leads to the development of thinner fingers. The fingers also tend to an asymptotic shape in the limit as surface tension tends to zero. Moreover, the asymptotic angle at the finger tip decreases as the viscosity ratio is reduced, apparently towards the zero angle (cusp) of the zero-viscosityratio solution.

The organization of the rest of the paper is as follows. In Sec. II, we present the equations of motion for the HeleShaw interface in a boundary integral formulation appropriate for the numerical method we use. In Sec. III, we describe the numerical method along with the main ideas that make it efficient. The numerical results are presented in Sec. IV. These results are divided in two main parts: the case when the surrounding fluid is inviscid and the effect of the surrounding-fluid viscosity. An analysis of the numerical errors is included in this section. Section IV also contains results showing that the qualitative features of the flow are insensitive to the initial position of the blob center with respect to the sink. Further discussion of the numerical results and conclusions are presented in Sec. V.

\section{THE GOVERNING EQUATIONS}

Typically in a Hele-Shaw cell, two viscous fluids are confined between two closely spaced parallel plates. Here, we consider the case of a blob of viscous fluid being sucked by an interior sink. The blob is surrounded by a less viscous fluid which fills the rest of the Hele-Shaw cell. For simplicity, we assume that the fluids are immiscible and incompressible with constant but differing viscosities. We use the subscripts 1 and 2 to refer to the fluids in the interior and exterior of the blob. The velocity field $\mathbf{u}_{j}$ of each fluid is given by Darcy's law,

$$
\mathbf{u}_{j}=-\frac{b^{2}}{12 \mu_{j}} \nabla p_{j}
$$

where $b$ is the cell gap, $\mu_{j}$ is the viscosity, and $p_{j}$ is the pressure. $M=b^{2} / 12 \mu_{j}$ is the fluid mobility. The incompressibility condition implies that $\nabla \cdot \mathbf{u}_{j}=0$ and therefore the pressure in each fluid satisfies Laplace's equation:

$$
\nabla^{2} p_{j}=0 .
$$


In other words, the interior and exterior fluids are potential. Consequently, the flow can be obtained from the dynamics of the free interface (blob boundary) between the two fluids. Denote the fluid interface by $\Gamma$. The interface motion is subject to the following conditions,

$$
\begin{aligned}
& {\left.[\mathbf{u} \cdot \hat{\mathbf{n}}]\right|_{\Gamma}=0,} \\
& {\left.[p]\right|_{\Gamma}=\tau \kappa,}
\end{aligned}
$$

where $[\cdot]$ denotes the jump across the interface taken as the difference of the interior minus the exterior quantity. Here, $\hat{\mathbf{n}}$ is the exterior unit normal to $\Gamma, \tau$ is the surface tension, and $\kappa$ is the interface mean curvature. The kinematic boundary condition (2.3) states that the normal component of the velocity field is continuous across the interface. This implies that particles on the interface remain there. The relation (2.4), known as the Laplace-Young boundary condition, gives an account of how the presence of surface tension modifies the pressure across the interface.

We assume that there is a point sink at the origin, inside the fluid blob. For large distances away from the sink, the velocity field tends to the simple radial flow:

$$
\mathbf{u}(\mathbf{x}) \rightarrow \mathbf{Q} \frac{\mathbf{x}}{|\mathbf{x}|^{2}}, \quad \text { as } \quad|\mathbf{x}| \rightarrow \infty .
$$

Here, $Q$ is the suction rate which is assumed constant and negative.

Let the interface $\Gamma$ be represented, at any instant $t$, by $(x(\alpha, t), y(\alpha, t))$, where $\alpha$ in $[0,2 \pi]$ defines a counterclockwise parametrization of $\Gamma$. Both $x$ and $y$ are periodic functions of $\alpha$. The interface governing equations can be put in a convenient form by introducing the complex position variable $z(\alpha, t)=x(\alpha, t)+i y(\alpha, t)$ and the complex conjugate velocity $W(\alpha, t)=u(\alpha, t)-i \mathrm{v}(\alpha, t)$. This interface velocity can be represented by a boundary integral plus the sink contribution as follows (for a derivation, see, e.g., Ref. 17)

$$
W=\frac{Q}{z(\alpha, t)}+\frac{1}{2 \pi i} \text { P.V. } \int_{0}^{2 \pi} \frac{\gamma\left(\alpha^{\prime}, t\right)}{z(\alpha, t)-z\left(\alpha^{\prime}, t\right)} d \alpha^{\prime},
$$

where the P.V. denotes the principal value integral. $\gamma$ is the (unnormalized) vortex sheet strength which measures the tangential velocity jump across the interface by

$$
\gamma=\left.s_{\alpha}[\mathbf{u}]\right|_{\Gamma} \cdot \hat{\mathbf{s}},
$$

where $s_{\alpha}=\sqrt{x_{\alpha}^{2}+y_{\alpha}^{2}}$, the subscript $\alpha$ denotes differentiation with respect to that variable, and $\hat{\mathbf{s}}$ is the unit tangential vector on $\Gamma . W(\alpha, t)$ gives, in complex form, the average velocity $(u, v)=\frac{1}{2}\left(\mathbf{u}_{1}+\mathbf{u}_{2}\right)$ on the interface and satisfies the boundary condition (2.5), provided $\gamma$ has zero mean. To close the system, an equation for $\gamma$ can be derived by using Eq. (2.1). We have that

$$
\begin{aligned}
\left(\nabla p_{1}-\nabla p_{2}\right) \cdot \hat{\mathbf{s}} & =\left(\frac{12 \mu_{2}}{b^{2}} \mathbf{u}_{2}-\frac{12 \mu_{1}}{b^{2}} \mathbf{u}_{1}\right) \cdot \hat{\mathbf{s}} \\
& =-\frac{12}{b^{2}}\left(\Delta \mu \mathbf{u}+\bar{\mu}\left(\mathbf{u}_{1}-\mathbf{u}_{2}\right)\right) \cdot \hat{\mathbf{s}},
\end{aligned}
$$

where $\Delta \mu=\mu_{1}-\mu_{2}, \bar{\mu}=\frac{1}{2}\left(\mu_{1}+\mu_{2}\right)$. Thus,

$$
\gamma=-s_{\alpha} \frac{\Delta \mu}{\bar{\mu}} \mathbf{u} \cdot \hat{\mathbf{s}}-s_{\alpha} \frac{b^{2}}{12 \bar{\mu}} \nabla\left(p_{1}-p_{2}\right) \cdot \hat{\mathbf{s}} .
$$

Using the dynamic boundary condition (2.4) and the complex conjugate velocity, we obtain the following equation for $\gamma$ :

$$
\gamma=2 \mathrm{~A}_{\mu} \operatorname{Re}\left\{z_{\alpha} W\right\}+S \kappa_{\alpha} .
$$

Here, $\mathrm{A}_{\mu}=\left(\mu_{1}-\mu_{2}\right) /\left(\mu_{1}+\mu_{2}\right)$ is the viscosity Atwood ratio and $S=\left(b^{2} / 12 \bar{\mu}\right) \tau$ is a scaled surface tension parameter. We nondimensionalize the equations of motion by taking the initial blob radius to be 1 and by setting $Q=-1$. Taking into account that there is freedom in selecting the tangential velocity at the interface, the evolutions equations can be written as

$$
\begin{aligned}
\bar{z}_{t}(\alpha, t)= & -\frac{1}{z(\alpha, t)}+\frac{1}{2 \pi i} \mathrm{P} . \mathrm{V} \cdot \int_{0}^{2 \pi} \frac{\gamma\left(\alpha^{\prime}, t\right)}{z(\alpha, t)-z\left(\alpha^{\prime}, t\right)} \\
& \times d \alpha^{\prime}+\mathrm{A}(\alpha, t) \frac{\bar{z}_{\alpha}(\alpha, t)}{\left|z_{\alpha}(\alpha, t)\right|} \\
\gamma(\alpha, t)= & 2 \mathrm{~A}_{\mu} \operatorname{Re}\left(-\frac{z_{\alpha}(\alpha, t)}{z(\alpha, t)}+\frac{z_{\alpha}(\alpha, t)}{2 \pi i} \mathrm{P} . \mathrm{V} .\right. \\
& \left.\times \int_{0}^{2 \pi} \frac{\gamma\left(\alpha^{\prime}, t\right)}{z(\alpha, t)-z\left(\alpha^{\prime}, t\right)} d \alpha^{\prime}\right)+S \kappa_{\alpha},
\end{aligned}
$$

where the bar denotes the complex conjugate. Here, $A(\alpha, t)$ is arbitrary and only determines the parametrization (frame) of the interface but does not affect its dynamics. For example, the frequently used Lagrangian frame is obtained by taking $A=\gamma /\left(2\left|z_{\alpha}\right|\right)$.

\section{THE NUMERICAL METHOD}

A spectrally accurate spatial discretization of Eqs. (2.11) and (2.12) can be achieved easily by computing the space derivatives with the pseudo-spectral approximation, i.e., using the fast Fourier transform (FFT). The principal value integral can also be computed with spectral accuracy by employing the alternate-point trapezoidal rule: ${ }^{18}$

$$
\text { P.V. } \int_{0}^{2 \pi} \frac{\gamma\left(\alpha^{\prime}\right)}{z\left(\alpha_{i}\right)-z\left(\alpha^{\prime}\right)} d \alpha^{\prime} \approx \sum_{\substack{j=0 \\(j-i) \text { odd }}}^{N-1} \frac{\gamma_{j}}{z_{i}-z_{j}} 2 h,
$$

where $N$ is the number of computational particles on the interface, $h=2 \pi / N$, and $\alpha_{i}=i h$ for $i=0, \ldots, N-1$. The quantities with subscripts are the corresponding discrete approximations. However, surface tension introduces a term $\left(\kappa_{\alpha}\right)$ with high-order derivatives that couple into the interface dynamics in a nonlinear and nonlocal manner. This leads to a high-order stability constraint (stiffness) for explicit timeintegration methods and makes implicit methods difficult to implement. Hou et al. ${ }^{10}$ have designed an efficient method to remove the high-order stability constraint. This is the method we use here. The method is based on a reformulation of the equations of motion in terms of variables more naturally related to the curvature and on the identification of the smallscale terms that contribute to the stiffness. The natural variables are the tangent angle to the interface $\theta$ and the arc- 
length metric $s_{\alpha}=\left|z_{\alpha}\right|$. In these two variables, the curvature has the simple form $\kappa=\theta_{\alpha} / s_{\alpha}$ and the evolution equations are given by

$$
\begin{aligned}
& s_{\alpha t}=T_{\alpha}-\theta_{\alpha} U, \\
& \theta_{t}=\frac{1}{s_{\alpha}}\left(U_{\alpha}+T \theta_{\alpha}\right),
\end{aligned}
$$

where $T$ and $U$ are the tangential and normal velocity, respectively. The stiffness is hidden at the small spatial scales of $U_{\alpha}$ in the $\theta$-equation. The leading order behavior of $U$ at small scales is given by ${ }^{10}$

$$
U(\alpha, t) \sim \frac{1}{2 s_{\alpha}} \mathcal{H}[\gamma](\alpha, t),
$$

where $\mathcal{H}$ is the Hilbert transform which is diagonalizable by the Fourier transform as $\hat{\mathcal{H}}[f]=-i \operatorname{sign}(k) \hat{f}$. The notation $f \sim g$ means that the difference between $f$ and $g$ is smoother than $f$ and $g$. Moreover, $\gamma$ is dominated by the surface tension term at small scales, that is,

$$
\gamma(\alpha, t) \sim S \kappa_{\alpha}=\left(\frac{\theta_{\alpha}}{s_{\alpha}}\right)_{\alpha} .
$$

Therefore,

$$
U(\alpha, t) \sim \frac{S}{2 s_{\alpha}} \mathcal{H}\left[\left(\theta_{\alpha} / s_{\alpha}\right)_{\alpha}\right](\alpha, t) .
$$

This dominant term at small scales simplifies if the arclength metric $s_{\alpha}$ is constant in space. This can be achieved by exploiting the freedom in selecting the tangential velocity. By letting

$$
T(\alpha, t)=\int_{0}^{\alpha} \theta_{\alpha^{\prime}} U d \alpha^{\prime}-\frac{\alpha}{2 \pi} \int_{0}^{2 \pi} \theta_{\alpha^{\prime}} U d \alpha^{\prime},
$$

$s_{\alpha}$ is maintained constant and equal to its mean at all times, i.e., $s_{\alpha}=L(t) / 2 \pi$, where $L(t)$ is the total length of the curve at time $t$. The equations of motion can now be written as

$$
\begin{aligned}
& L_{t}=-\int_{0}^{2 \pi} \theta_{\alpha^{\prime}} U d \alpha^{\prime}, \\
& \theta_{t}=\frac{S}{2}\left(\frac{2 \pi}{L}\right)^{3} \mathcal{H}[\theta]_{\alpha \alpha \alpha}+P,
\end{aligned}
$$

where $P$ represents lower-order terms at small spatial scales. $L$ can be updated by an explicit method as Eq. (3.8) is free of stiffness. To remove the high-order stiffness it is sufficient to discretize implicitly the leading order term in Eq. (3.9) and treat the lower order term $P$ explicitly. This gives a linear time-step stability constraint, i.e., $\Delta t \leqslant C h$, where $\Delta t$ is the time-step size. Moreover, because of its constant coefficients, the implicit term can be easily inverted by using FFT. Here, we use the following fourth-order explicit/implicit method studied by Ascher et al.: ${ }^{19}$

$$
\begin{aligned}
\frac{1}{\Delta t}( & \left.\frac{25}{12} \theta^{n+1}-4 \theta^{n}+3 \theta^{n-1}-\frac{4}{3} \theta^{n-2}+\frac{1}{4} \theta^{n-3}\right) \\
= & \frac{S}{2}\left(\frac{2 \pi}{L^{n+1}}\right)^{3} \mathcal{H}\left[\theta^{n+1}\right]_{\alpha \alpha \alpha}+4 P^{n}-6 P^{n-1} \\
& +4 P^{n-2}-P^{n-3}
\end{aligned}
$$

Here $L$ is updated first using a fourth-order explicit AdamsBashforth multi-step scheme to obtain $L^{n+1}$ before computing $\theta^{n+1}$ via FFT.

Note that, at each time step, $\gamma$ has to be obtained from (2.12) to compute the velocities. It has been shown ${ }^{20}$ that the Fredholm integral equation for $\gamma$ has a globally convergent Neumann series. We solve for $\gamma$ by fixed point iteration, accelerated by constructing a fourth-order extrapolated initial guess from previous time steps. It typically takes a few iterations to obtain a convergent solution for $\gamma$ when the interface is relatively smooth. The overall method is fourth order in time and spectrally accurate in space.

\section{NUMERICAL RESULTS}

We consider an initially circular blob of viscous fluid surrounded by less viscous fluid. The center of the initial blob is at $(0,-0.1)$ and the sink is placed at the origin. Our numerical results focus on the regularizing effect of surface tension, past the $S=0$ singularity time, and on the limiting behavior of the interface as surface tension tends to zero. The results are divided in two main parts. First, we present the case where the viscosity of the surrounding fluid is negligible, which corresponds to $\mathrm{A}_{\mu}=1$. In the second part, we investigate the additional effect that nonzero viscosity in the surrounding fluid has on the interface dynamics, i.e., $\mathrm{A}_{\mu}$ $<1$.

All the computations presented here are performed in 64-bit arithmetic (standard double precision). Krasny filtering ${ }^{21}$ is used to prevent the spurious growth of the highfrequency components of the round-off error. This nonlinear numerical filter is implemented by setting to zero all the Fourier modes of the solution whose magnitudes are below a certain level. In our computations we set this filter level to be $10^{-12}$. The number $N$ of uniformly spaced points along the interface is chosen so that all the Fourier modes of the tangent angle $\theta(\alpha, t)$ are well resolved. Initially, we use $N$ $=2048$ for most of the computations. The time step $\Delta t$ is selected so that decreasing it further would not produce any appreciable difference within plotting resolution in the curvature of the interface. We double $N$ as soon as the magnitude of the highest frequency mode of $\theta(\alpha, t)$ is greater than the filter level. Very small values of $\Delta t$ are required to compute accurately the interface motion as it approaches the sink. At the latest stage of the motion, the number of grid points typically increases to $N=8192$ or $N=16384$ and $\Delta t$ $=2 \times 10^{-7}$. A detailed resolution study is presented later in this section. 


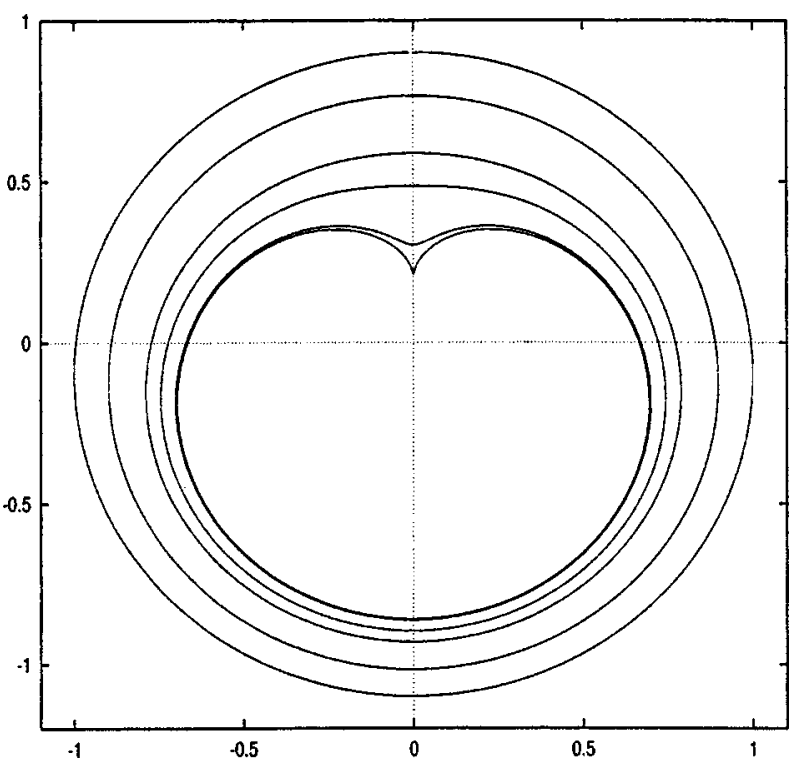

FIG. 1. Evolution and collapse of the interface for the initially circular blob centered at $(0,-0.1)$ being sucked by a point sink at the origin. $S=0$ and $\mathrm{A}_{\mu}=1$. The curves, from the outer perimeter inwards, correspond to the times $t=0,0.1,0.2,0.24,0.28$, and 0.2842 . The cusp is formed approximately at $t=0.2842$. The computation was performed using $N=2048$ and $\Delta t=1 \times 10^{-5}$ in the Lagrangian frame.

\section{A. Development of the interface for $A_{\mu}=1$}

\section{Zero-surface-tension cusp formation}

In the absence of surface tension, an initially circular blob whose center is not at the sink develops a $\frac{3}{2}$-power cusp singularity in finite time. Figure 1 shows the evolution and collapse of the fluid interface for $A_{\mu}=1$ and $S=0$. The cusp occurs at approximately $t_{c}=0.2842$ and is located at $(0$, $0.2305)$ for this particular case where the blob is initially centered at $(0,-0.1)$. To resolve well the interface up to times very close to the formation of the cusp, we take advantage of the particle clustering characteristic of the Lagrangian frame. That is, for this special case $(S=0)$, we discretize directly Eqs. (2.11) and (2.12) with $A=\gamma /\left(2\left|z_{\alpha}\right|\right)$ using the pseudo-spectral approximation for the derivatives and the alternate-point trapezoidal rule (3.1) for the singular integral. An explicit fourth-order Adams-Bashforth scheme is used to integrate in time.

\section{The regularizing effect of surface tension}

The presence of surface tension regularizes the cusped flow in a very special way. Figure 2 shows the fluid interface at different times for $S=0.01$. A smooth finger with a rounded tip develops past the zero-surface-tension breakdown time $t_{c}=0.2842$. As the interface gets closer to the sink, the finger evolves rapidly into a wedge. We stop the computation when the distance of the finger tip to the sink is $5.9848 \times 10^{-3}$. We use $N=8192$ and $\Delta t=2 \times 10^{-7}$ for the last stage of the computation. The formation of the wedge as the interface approaches the sink is consistent with the results reported by Nie and Tian ${ }^{12}$ for the two values of surface tension they computed.

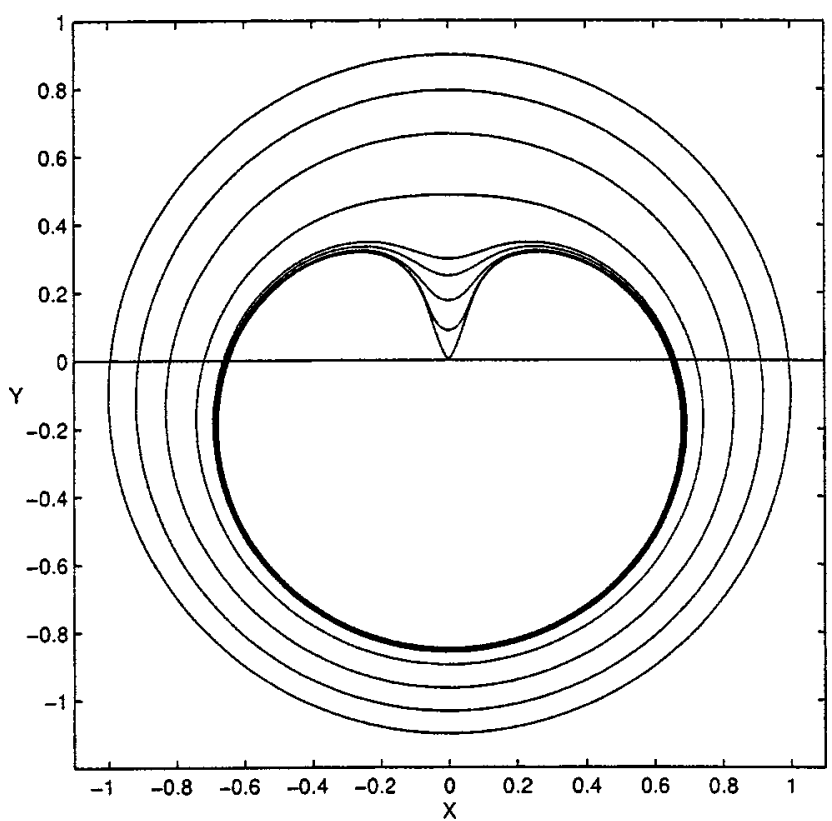

FIG. 2. Evolution of the initially circular fluid blob for $S=0.01$ and $\mathrm{A}_{\mu}$ $=1$. The curves, from the outer perimeter inwards, correspond to the times $t=0,0.080,0.160,0.240,0.284,0.290,0.296,0.300$, and 0.301419 . The distance of the tip of the finger to the sink, for the last computed interface $(t=0.301419)$, is $5.9848 \times 10^{-3}$ and the tip curvature is $-180.37 . N$ $=8192$ and $\Delta t=2 \times 10^{-7}$ for the last stage of the motion.

We decrease now surface tension to the value $S=4$ $\times 10^{-4}$. A time sequence of the interface evolution for this small surface tension is presented in Fig. 3. The interface passes smoothly the zero-surface-tension cusp time $t_{c}$. Soon after $t_{c}$, we observe the appearance of an almost straight

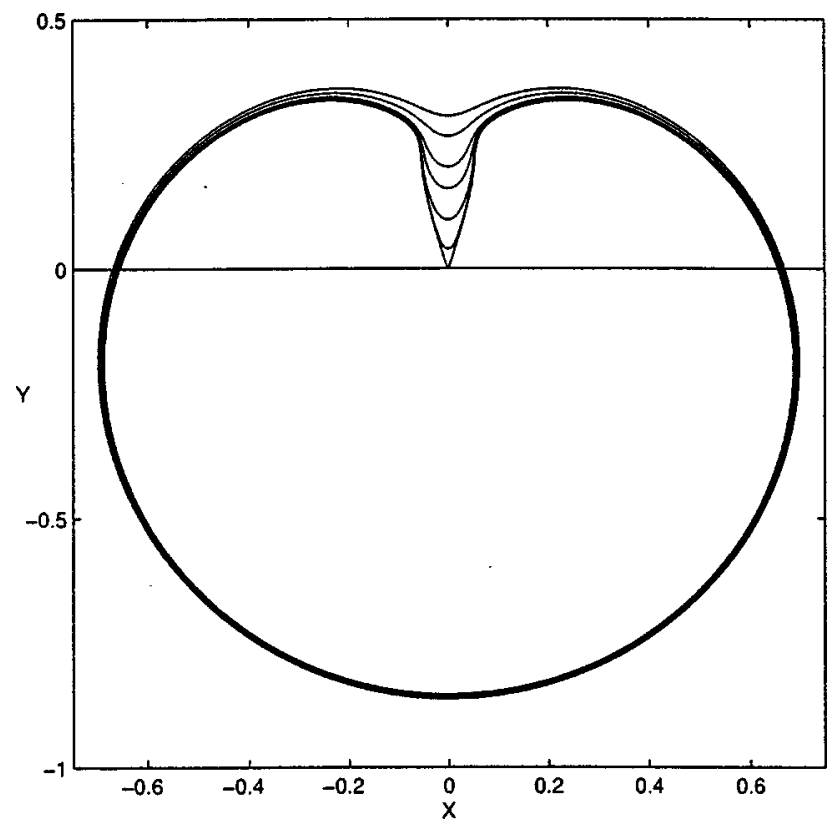

FIG. 3. Evolution of the initially circular fluid blob for $S=4 \times 10^{-4}$ and $\mathrm{A}_{\mu}=1$. The interface is plotted at times around $t_{c}=0.2842$ and well past it. From the the outer perimeter inwards, the curves correspond to the times $t$ $=0.280,0.284,0.288,0.290,0.292,0.293$, and 0.2932. $N=16384$ and $\Delta t$ $=2 \times 10^{-7}$ for the last stage of the motion. At $t=0.2932$, the distance of the wedge tip to the sink is $8.9 \times 10^{-4}$ and the tip curvature is -1945.26 . 


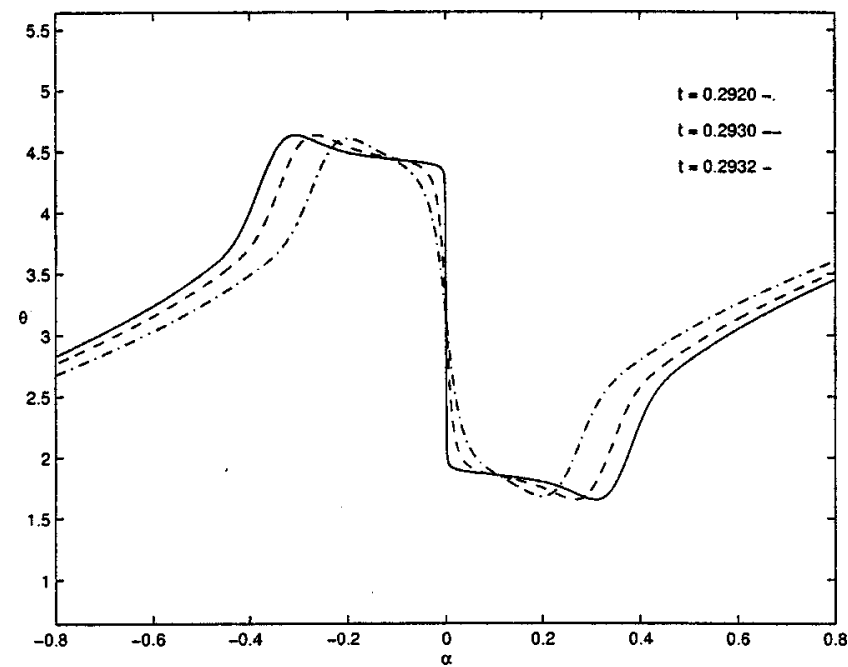

FIG. 4. Behavior of the tangent angle $\theta(\alpha, t)$ around the finger tip $(\alpha=0)$ as the interface is about to collapse, for $S=4 \times 10^{-4}$ and $\mathrm{A}_{\mu}=1$. The tangent angle, plotted against the parametrization variable $\alpha$ at the times $t$ $=0.2920,0.2930$, and 0.2932, appears to develop a discontinuity. $N$ $=16384$ and $\Delta t=2 \times 10^{-7}$.

finger that begins to bulge but is quickly drawn into the sink forming a wedge. Note that the top of this finger is narrower than the one corresponding to the larger surface tension $S$ $=0.01$ (Fig. 2). A look at the tangent angle $\theta(\alpha, t)$ around the finger tip for $S=4 \times 10^{-4}$, shown in Fig. 4, strongly suggests the formation of a corner when the interface touches the sink. The tangent angle $\theta$ appears to develop a discontinuity at the finger tip $(\alpha=0)$, precisely when the Hele-Shaw solution breaks down. The computations of Nie and Tian, ${ }^{12}$ for another type of initial data, also suggest this breakdown scenario. The formation of the wedge and the tip corner seem to be generic for this type of flow.

Smaller values of surface tension reveal new features in the interface evolution. Figure 5 shows the interface shape for $S=5 \times 10^{-5}$. The finger clearly bulges and develops a well-defined neck before it becomes a wedge. It is interesting to note that this neck appears at a height close to that of the zero-surface-tension cusp. It is conceivable that the formation of the neck and the bulging of the finger are due to the influence of the zero-surface-tension singularity. In fact, a look at the curvature shown at Fig. 6(a) for the time $t$ $=0.2860$, which is very close to $t_{c}=0.2842$, shows already the appearance of two symmetric spikes corresponding to the location of the neck. The behavior of the interface curvature at subsequent times is shown in Figs. 6(c) and 6(d). Note in particular that the curvature grows almost ten times in magnitude from $t=0.2916$ [Fig. 6(c)] to $t=0.2918$ [Fig. 6(d)]. The sharp and large spike at $\alpha=0$ is an indication of the corner singularity forming as the tip of the wedge touches the sink. We use $N=16384$ and $\Delta t=2 \times 10^{-7}$ to resolve accurately this large curvature motion. At $t=0.2918$, the distance of the wedge tip to the sink is $r=2.92 \times 10^{-3}$ and the tip curvature is $\kappa=-1371.11$. The sink pressure $-\log r$ dominates the surface tension pressure $S \kappa$.
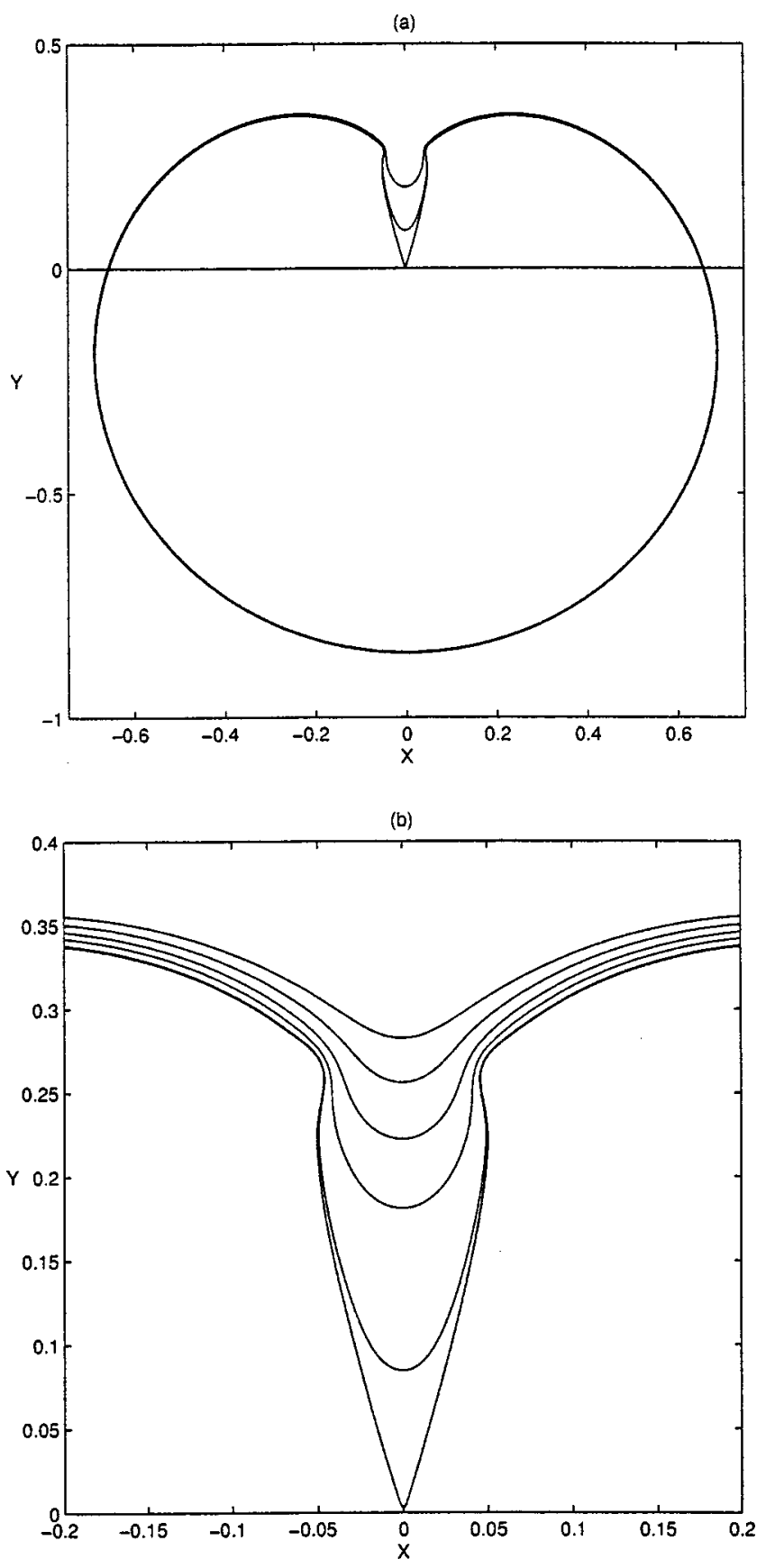

FIG. 5. Evolution of the initially circular fluid blob past $t_{c}$, for $S=5$ $\times 10^{-5}$ and $\mathrm{A}_{\mu}=1$. (a) The interface plotted at $t=0.2880,0.2900$, and 0.29181 . (b) A close-up of the interface finger at the times $t=0.2840$, $0.2860,0.2880,0.2900$, and $0.29181 . N=16384$ and $\Delta t=2 \times 10^{-7}$ for the last stage of the motion. At $t=0.29181$, the distance of the wedge tip to the sink is $2.92 \times 10^{-3}$ and the tip curvature is -1371.11 .

\section{The interface limiting behavior as $S \rightarrow 0$}

We investigate now the interface limiting behavior before and past $t_{c}$. We present numerical evidence to show that an asymptotic corner angle is selected in the limit as surface tension tends to zero when the finger tip is about to reach the sink. The computations presented here also suggest that the vanishing surface tension solution is singular at the finger neck.

To obtain information on the behavior of the wedge angle in the limit as surface tension tends to zero, we com- 

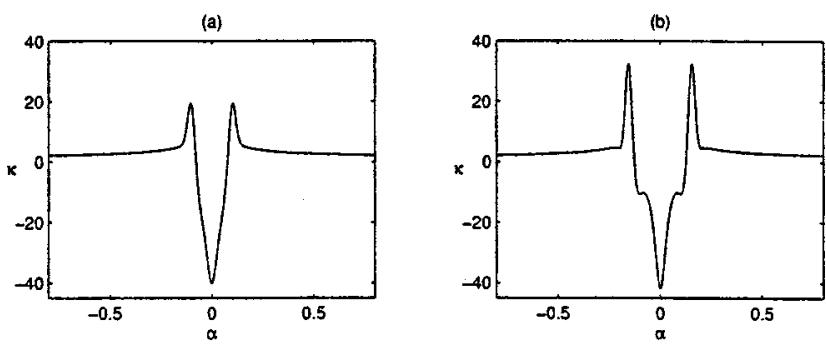

(c)
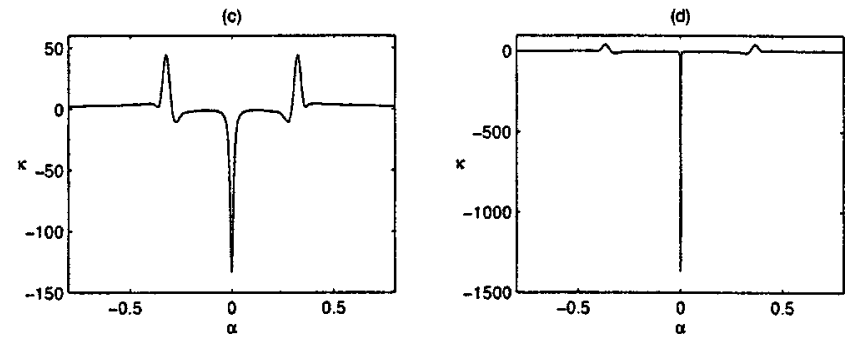

FIG. 6. Interface curvature $\kappa(\alpha, t)$ versus $\alpha$ around the finger tip $(\alpha=0)$ at different times past $t_{c}$ for $S=5 \times 10^{-5}$ and $\mathrm{A}_{\mu}=1$. (a) $t=0.2860$. (b) $t$ $=0.2880$. (c) $t=0.2916$. (d) $t=0.29181 . N=16384$ and $\Delta t=2 \times 10^{-7}$ were used to resolve the largest curvature (d).

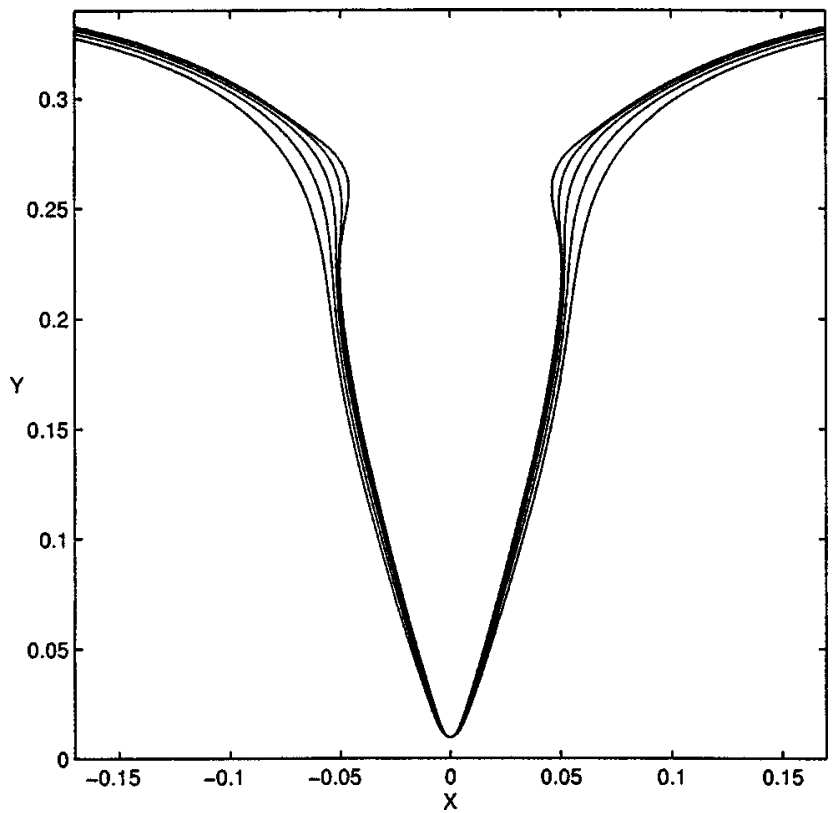

FIG. 7. Comparison of the interface finger for a sequence of surface tensions with $\mathrm{A}_{\mu}=1$. From the outer curve inwards, the fingers correspond to the surface tension values $S=8 \times 10^{-4}, 4 \times 10^{-4}, 2 \times 10^{-4}, 1 \times 10^{-4}$, and $5 \times 10^{-5}$. Each interface is plotted when the tip of the finger reaches the fixed level $y=0.01$ at $x=0 . N=16384$ and $\Delta t=2 \times 10^{-7}$.

TABLE I. Change in the finger widths as surface tension is decreased for $\mathrm{A}_{\mu}=1$. The first column shows the height level at which the fingers are compared. Columns 2-5 give the difference $\Delta(S, S / 2)$ between the width of the finger corresponding to a surface tension $S$ and that corresponding to $S / 2$.

\begin{tabular}{ccccc}
\hline \hline & $\Delta(0.0008$, & $\Delta(0.0004$, & $\Delta(0.0002$, & $\Delta(0.0001$, \\
$y$ & $0.0004)$ & $0.0002)$ & $0.0001)$ & $0.00005)$ \\
\hline 0.1 & $1.63 \times 10^{-3}$ & $1.17 \times 10^{-3}$ & $8.96 \times 10^{-4}$ & $6.62 \times 10^{-4}$ \\
0.06 & $1.24 \times 10^{-3}$ & $9.14 \times 10^{-4}$ & $6.98 \times 10^{-3}$ & $5.39 \times 10^{-4}$ \\
0.02 & $5.99 \times 10^{-4}$ & $4.57 \times 10^{-4}$ & $3.45 \times 10^{-4}$ & $2.47 \times 10^{-4}$ \\
\hline \hline
\end{tabular}

TABLE II. The angle of the wedge (in radiants) for a decreasing set of surface tensions. The variation (third column) is the difference between consecutive angles, corresponding to surface tensions $S$ and $2 S$.

\begin{tabular}{ccc}
\hline \hline$S$ & Wedge angle & Variation \\
\hline $8 \times 10^{-4}$ & 0.67459 & $\ldots$ \\
$4 \times 10^{-4}$ & 0.65719 & 0.0174 \\
$2 \times 10^{-4}$ & 0.64399 & 0.0132 \\
$1 \times 10^{-4}$ & 0.63660 & 0.0074 \\
$5 \times 10^{-5}$ & 0.63359 & 0.0030 \\
\hline \hline
\end{tabular}

pare the interfaces for a set of decreasing values of surface tension. Since the velocity of the interface depends on surface tension, we compare the interfaces when their finger tips reach the same level above the sink rather than at a fixed time. As surface tension is reduced, the finger tip reaches the given level faster. Figure 7 provides some indication of the asymptotic trend of the fingers as surface tension is successively halved from $S=8 \times 10^{-4}$ to $S=5 \times 10^{-5}$. The fixed level is $y=0.01$ so that the finger tips are very close to the sink. As surface tension is decreased, the fingers develop a neck at about $y=0.27$. However, away from the neck, the finger width changes very little. More precisely, as surface tension is reduced, the change in the finger width decreases. Table I gives the difference $\Delta(S, S / 2)$ between the width of the finger corresponding to a surface tension $S$ and that corresponding to $S / 2$ at three different levels. It is observed that $\Delta(S, S / 2)$ decreases as surface tension is reduced. The fingers are converging to an asymptotic shape. Table II suggests that an asymptotic angle is selected for the wedge as it touches the sink. The difference between consecutive angles

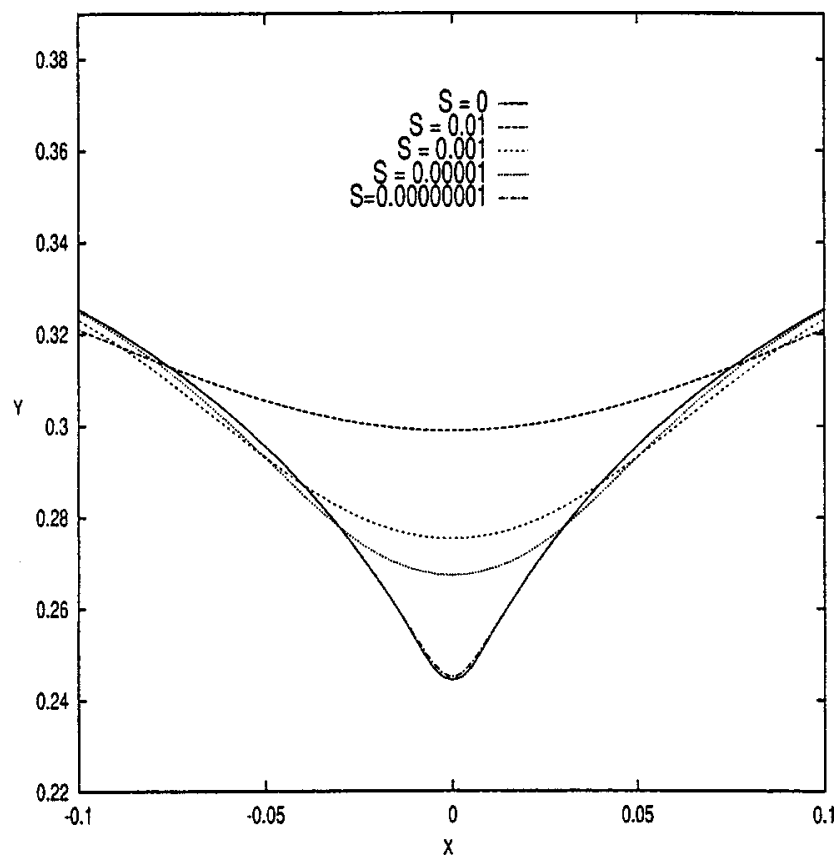

FIG. 8. Limiting behavior of the interface before $t_{c}=0.2842$ for $\mathrm{A}_{\mu}=1$. This figure shows a close-up picture of the interface around $x=0$ for a set of surface tension values, decreasing from top to bottom, and plotted at time $t=0.2840$. The zero-surface-tension solution is also shown. $N=4096$ and $\Delta t=5 \times 10^{-5}$. 
(a)

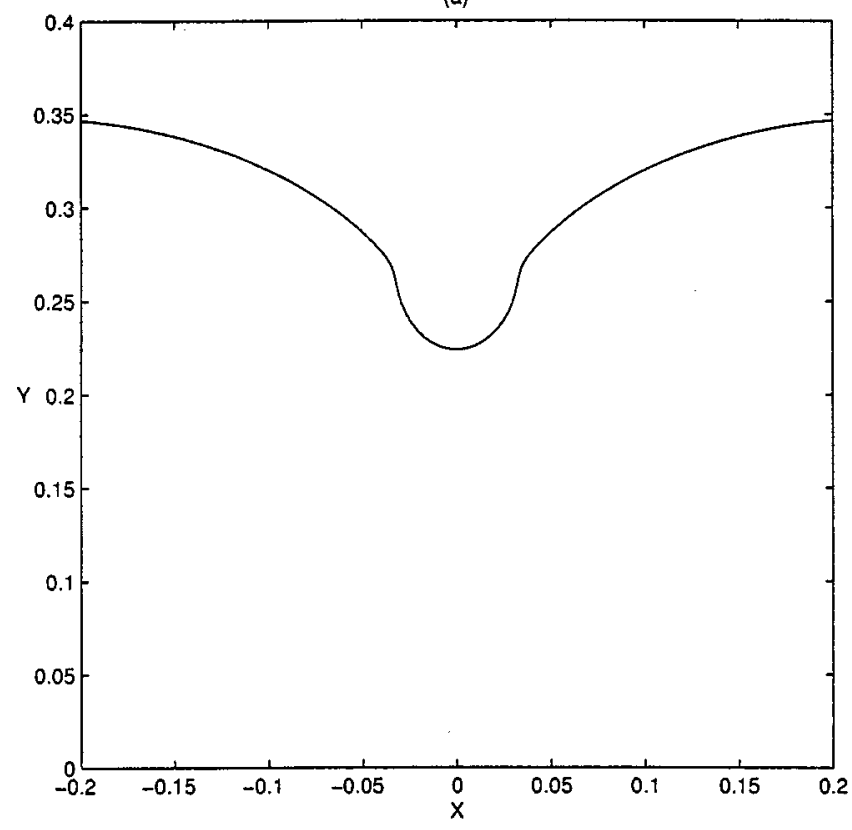

(b)

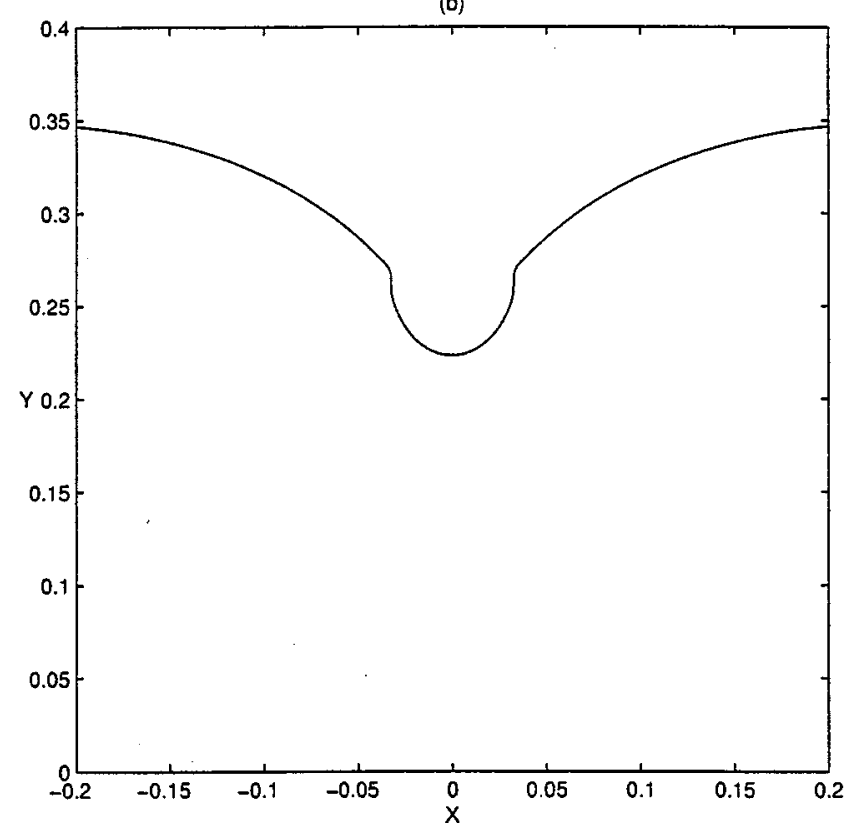

FIG. 9. Close-up of the interface around $x=0$ at $t=0.2857$ (just past $t_{c}$ ) for $\mathrm{A}_{\mu}=1$. The interfaces correspond to (a) $S=10^{-5}$ and (b) $S=5 \times 10^{-6} . N$ $=8192$ and $\Delta t=5 \times 10^{-6}$.

(corresponding to surface tensions $S$ and $2 S$ ) decreases as surface tension is reduced. Note also that there is an asymptotic time at which the limiting wedge reaches the sink.

While the asymptotic trend of the wedge angle is clear, the limiting behavior of the interface in the vicinity of the neck is not obvious. More precisely, we would like to find the form of the continuation solution selected in the limit as surface tension tends to zero for any fixed time past $t_{c}$ but before the time at which the limiting wedge reaches the sink. As expected, before $t_{c}$, the interface converges to the smooth zero-surface-tension solution as $S \rightarrow 0$. This is illus-
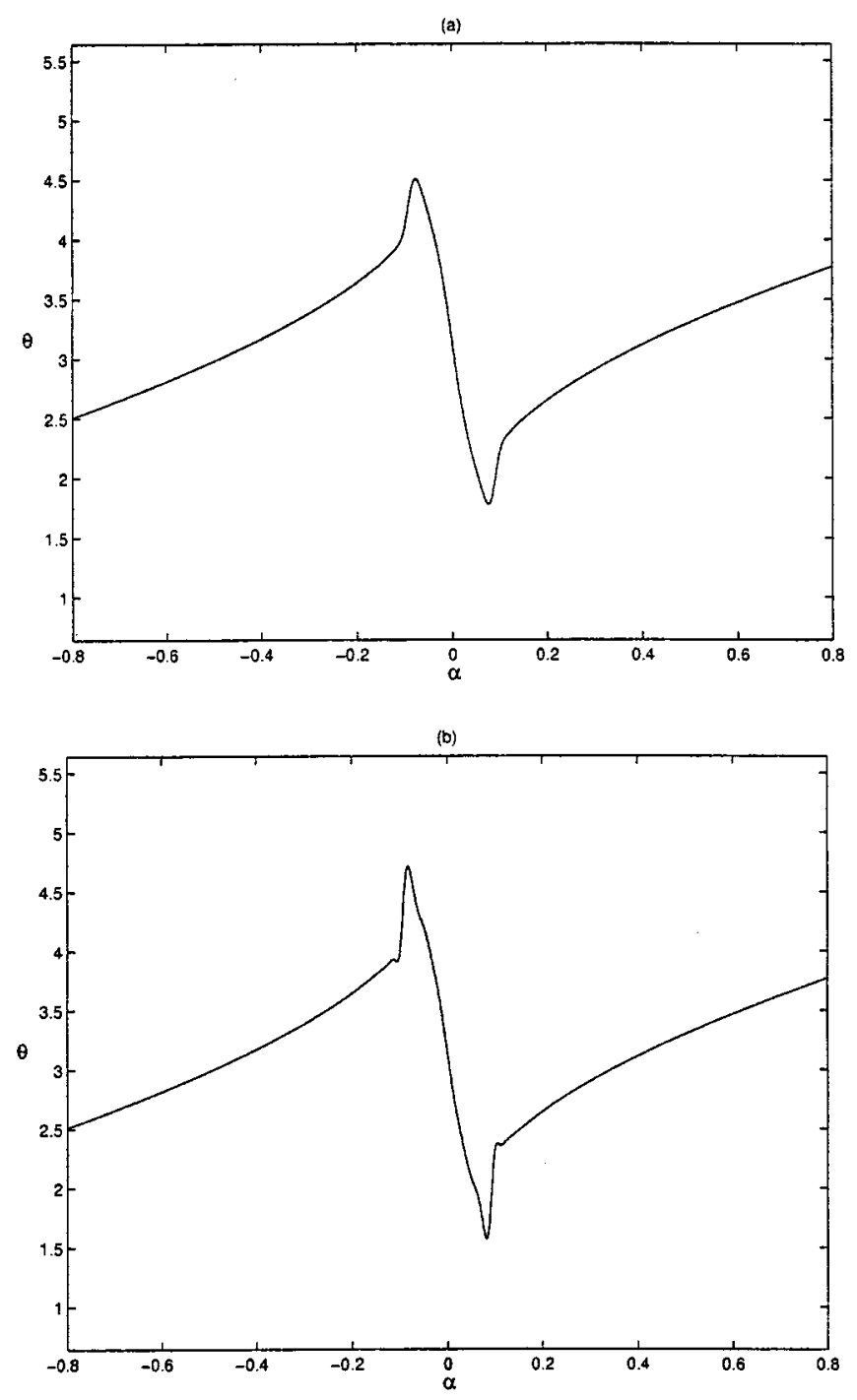

FIG. 10. The tangent angle $\theta(\alpha, t)$ versus $\alpha$ around the finger tip ( $\alpha=0)$ at $t=0.2857$ for $\mathrm{A}_{\mu}=1$. The plots correspond to (a) $S=10^{-5}$ and (b) 5 $\times 10^{-6} . N=8192$ and $\Delta t=5 \times 10^{-6}$.
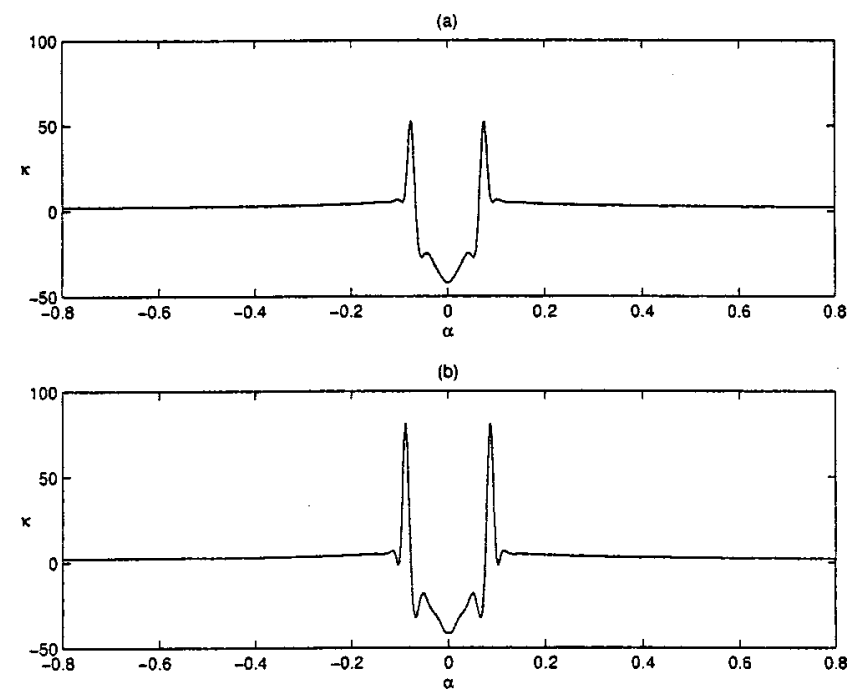

FIG. 11. The curvature $\kappa(\alpha, t)$ versus $\alpha$ around the finger tip $(\alpha=0)$ for $\mathrm{A}_{\mu}=1$ and $S=5 \times 10^{-6}$, at (a) $t=0.2850$ and (b) $t=0.2855$. $N=8192$ and $\Delta t=5 \times 10^{-6}$. 
(a)

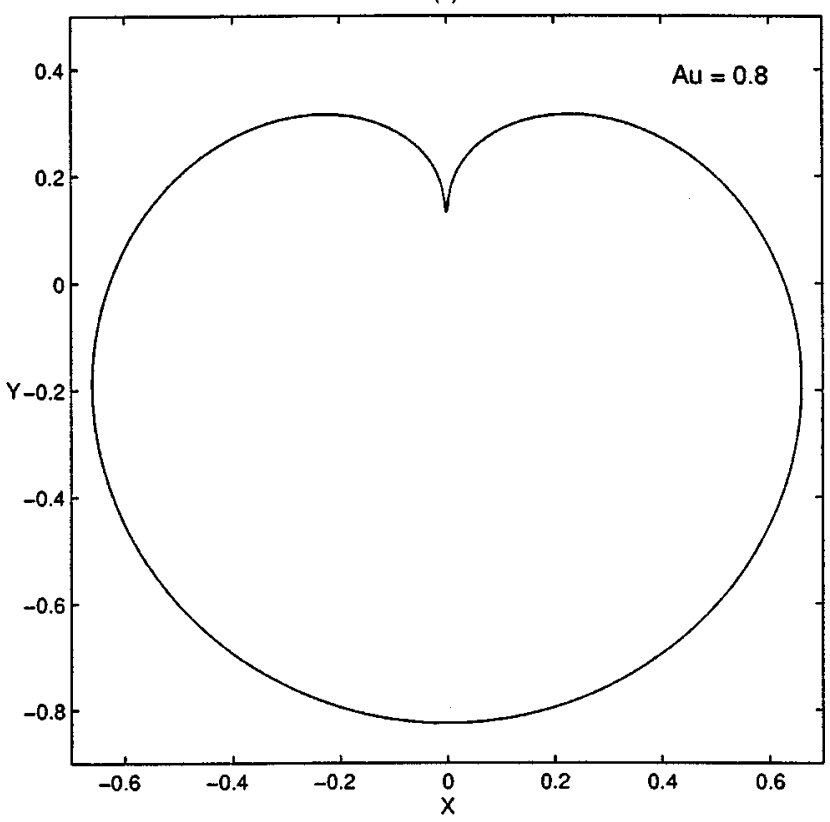

(b)

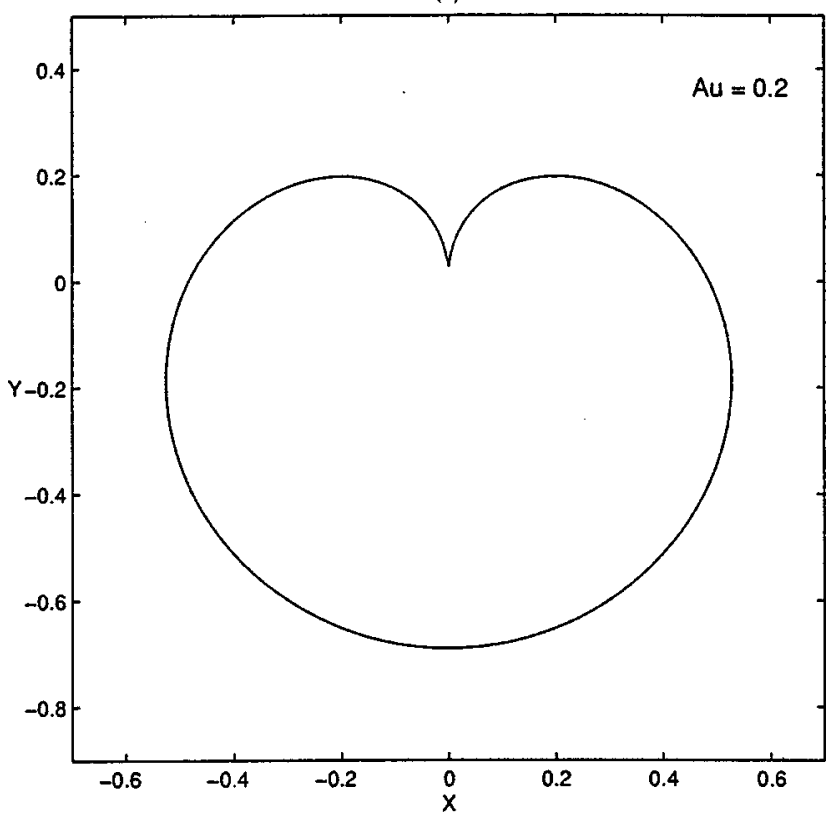

FIG. 12. Collapse of the interface in the absence of surface tension. (a) $\mathrm{A}_{\mu}=0.8$. The cusp is formed approximately at $t_{c}=0.3070$. (b) $\mathrm{A}_{\mu}=0.2$. The cusp is formed approximately at $t_{c}=0.3809$. The computations were performed using $N=2048$ and $\Delta t=1 \times 10^{-5}$ in the Lagrangian frame.

trated in Fig. 8 which shows a close-up picture of the interface for a set of decreasing surface tensions at a time $(t$ $=0.2840$ ) slightly before $t_{c}$. The zero-surface-tension solution is also shown in Fig. 8.

We observe a very different behavior past $t_{c}$. Figure 9 presents close-up pictures of the interface finger developing just past $t_{c}$. The interfaces correspond to the surface tension values $S=1 \times 10^{-5}$ and $S=5 \times 10^{-6}$, plotted at $t=0.2857$. Note that the width of the small finger is approximately the same for both surface tensions. But a more pronounced neck with two corners can be observed for $S=5 \times 10^{-6}$ in Fig.
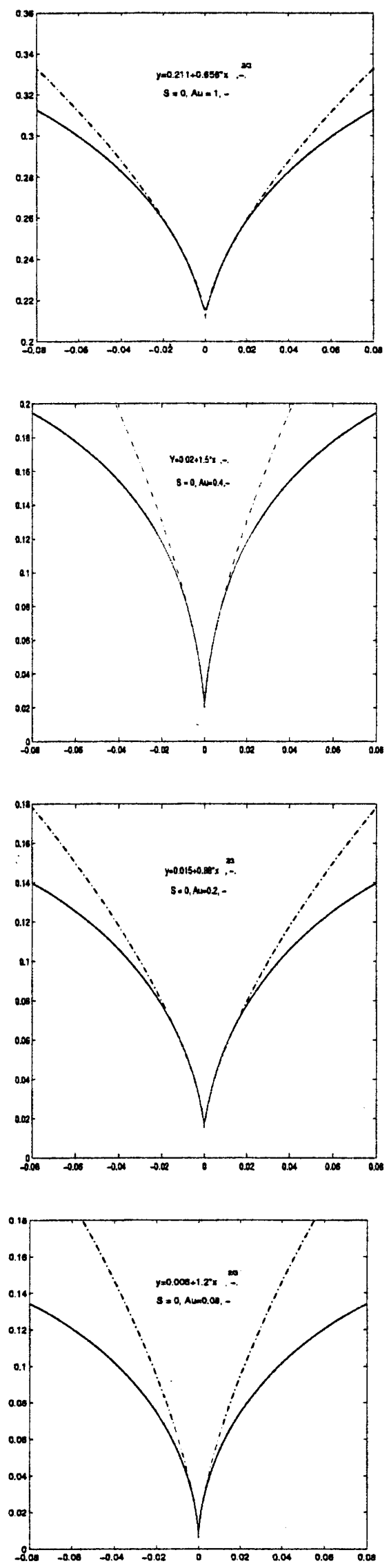

FIG. 13. Zero-surface-tension cusps and $\frac{3}{2}$-power fitting curves for different values of the viscosity Atwood ratio, shown at the approximate breakdown time $t_{c}$. (a) $\mathrm{A}_{\mu}=1.0$ at $t_{c}=0.2842$. (b) $\mathrm{A}_{\mu}=0.4$ at $t_{c}=0.3745$. (c) $\mathrm{A}_{\mu}$ $=0.2$ at $t_{c}=0.3807$. (d) $\mathrm{A}_{\mu}=0.08$ at $t_{c}=0.40135$. The computations were performed using $N=2048$ and $\Delta t=1 \times 10^{-5}$ in the Lagrangian frame. 
(a)

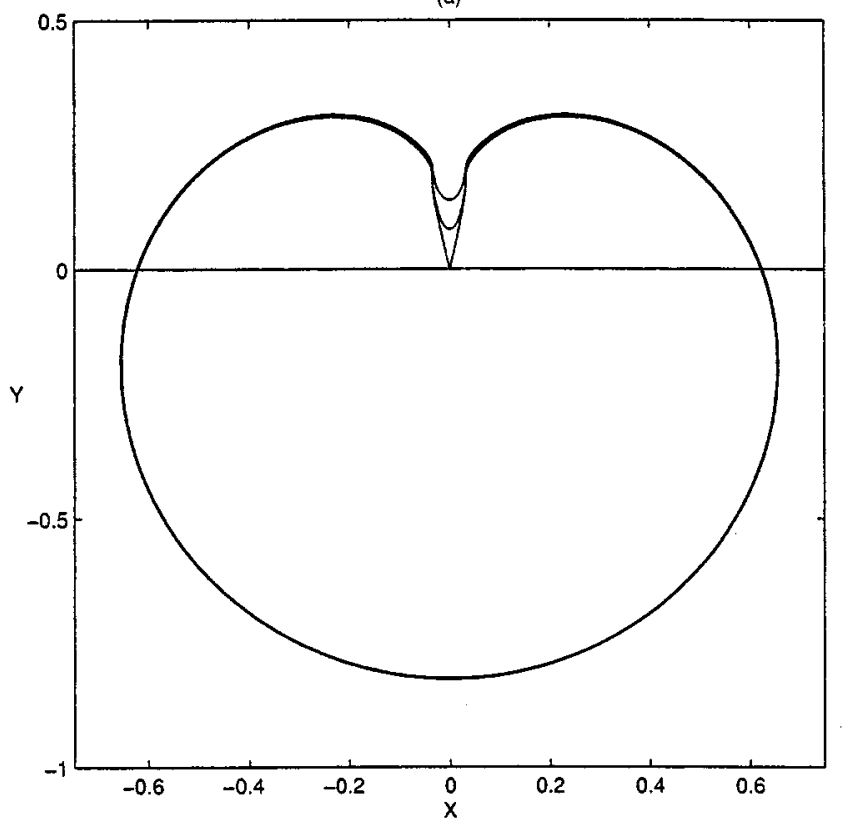

(b)

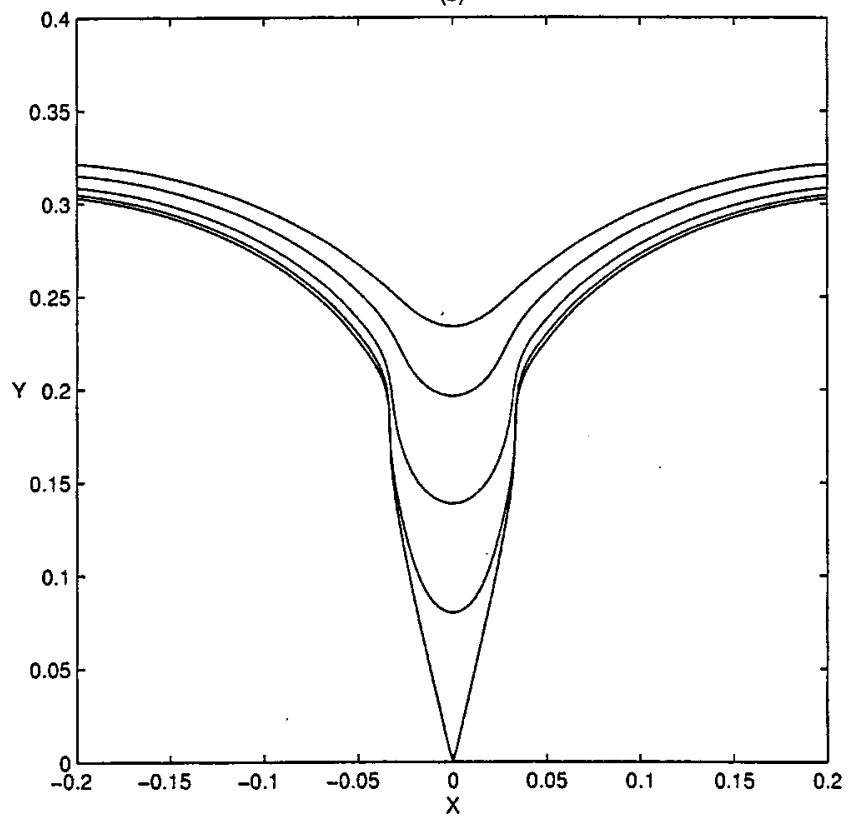

FIG. 14. Evolution of the initially circular fluid blob past $t_{c}$, for $S=5$ $\times 10^{-5}$ and $\mathrm{A}_{\mu}=0.8$. (a) The interface plotted at $t=0.3106,0.3126$, and 0.3136 . (b) A close-up of the interface finger at the times $t=0.304,0.3076$, 0.3106, 0.3126, and 0.3136. $N=16384$ and $\Delta t=2 \times 10^{-7}$ for the last stage of the motion.

9(b). Unfortunately, well-resolved computations for surface tensions smaller that this value are extremely difficult due interface singular behavior and to growth of the round-off error noise. Nevertheless, the nonsmooth transition observed for the previous values of surface tension hints a possible singularity formation in the limit. Indeed, a look at the tangent angle $\theta(\alpha, t)$ in Fig. 10 provides further indication of a singularity scenario in the limit as $S \rightarrow 0$. The two spikes in $\theta$ correspond to the finger neck. While the tangent angle changes smoothly around the finger tip, two small kinks observed for $S=5 \times 10^{-6}$ mark an almost discontinuous transi-
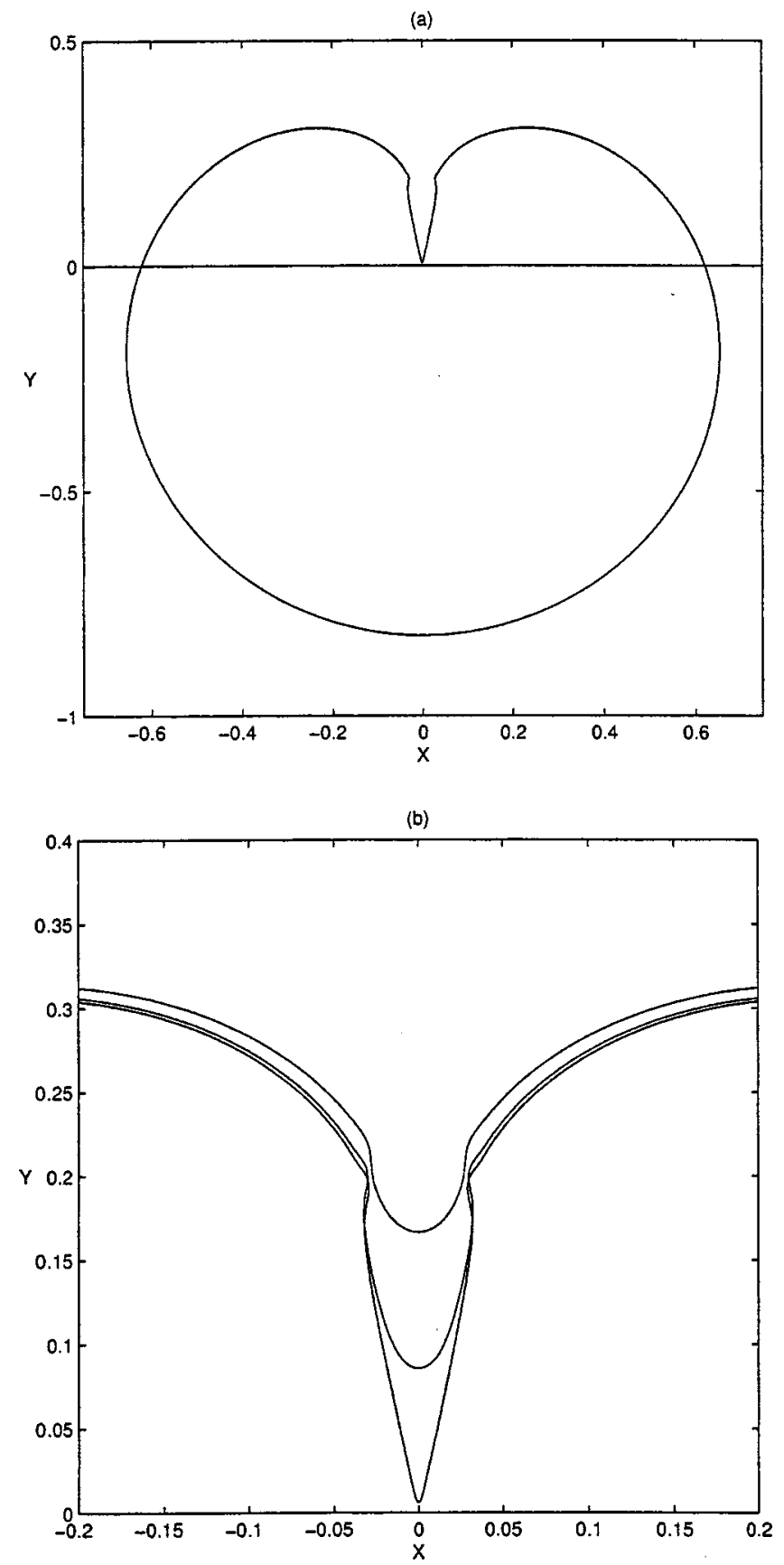

FIG. 15. Evolution of the initially circular fluid blob past $t_{c}$, for $S=1$ $\times 10^{-5}$ and $\mathrm{A}_{\mu}=0.8$. (a) The interface plotted at $t=0.31222$. (b) $\mathrm{A}$ close-up of the interface finger at the times $t=0.3080,0.3111$, and 0.31222 . $N=32768$ and $\Delta t=1 \times 10^{-7}$ for the last stage of the motion.

tion in $\theta$ at the finger neck. We use $N=8192$ and $\Delta t=5$ $\times 10^{-5}$ to resolve accurately both $\theta$ and the curvature for this case. Figure 11 shows the curvature near the finger tip at two very close times for $S=5 \times 10^{-6}$. There is a rapid growth of the curvature at the neck. A comparison with the curvature corresponding to $S=5 \times 10^{-5}$ [Fig. 6(a)] shows the singular trend of the interface limiting behavior. Although the numerical evidence is somewhat limited and further study is required for smaller values of surface tension, we conjecture that the neck will asymptote to corners in the limit as $S$ $\rightarrow 0$. 


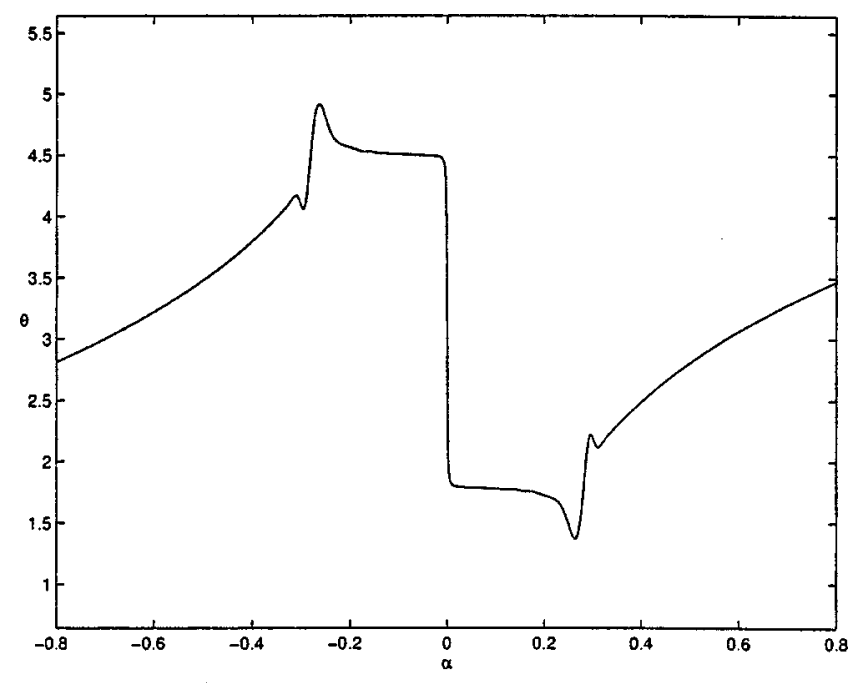

FIG. 16. Tangent angle $\theta(\alpha, t)$ versus $\alpha$ around the finger tip at $t$ $=0.31222$ for $S=1 \times 10^{-5}$ and $\mathrm{A}_{\mu}=0.8 . N=32768$ and $\Delta t=10^{-7}$.

\section{B. $A_{\mu}<1$ : The effect of the surrounding-fluid viscosity}

\section{Zero-surface-tension cusp formation}

Very little is known for the Hele-Shaw flow with suction when $\mathrm{A}_{\mu}<1$, i.e., for the two-phase (Muskat) problem, even if surface tension is neglected. We first present a series of computations for several Atwood ratios in the absence of surface tension. These computations for $S=0$ are performed in the Lagrangian frame as explained in Sec. IV A 1. Figure 12 shows the breakdown of the Hele-Shaw solution for $\mathrm{A}_{\mu}$ $=0.8$ and $\mathrm{A}_{\mu}=0.2$. The viscosity of the exterior fluid alone does not prevent the formation of cusps in the interface. Note that the breakdown times occur later than that for the $\mathrm{A}_{\mu}$ $=1$ flow. As a result, more interior fluid gets sucked as $\mathrm{A}_{\mu}$ decreases. Figure 13 presents close-up plots of the zerosurface-tension cusps for $\mathrm{A}_{\mu}=1,0.4,0.2$, and 0.08. As the fitting curves demonstrate, the $\frac{3}{2}$-power cusp singularity appears to be very generic. In all these cases the cusps are formed before the interface reaches the sink. However, as $\mathrm{A}_{\mu}$ is reduced, the cusps develop closer and closer to the sink. It may be thought that for sufficiently small $\mathrm{A}_{\mu}$ the cusp singularity will be formed only when the interface touches the sink. Nevertheless, at least for $\mathrm{A}_{\mu}$ as small as 0.01 , we find no evidence of this. The cusp still forms before the interface reaches the sink. Apparently, only for $\mathrm{A}_{\mu}=0$ the cusp occurs right at the sink.

\section{Surface tension and large viscosity ratio}

We consider now the flow for $\mathrm{A}_{\mu}=0.8$ with surface tension $S=5 \times 10^{-5}$. Figure 14 shows the interface at different times as it evolves. Just as in the $\mathrm{A}_{\mu}=1$ case, a finger develops past $t_{c}$ and evolves into a wedge as it is drawn into the sink. This particular value of surface tension was also considered for $\mathrm{A}_{\mu}=1$ (see Fig. 5). A comparison of these two cases, $\mathrm{A}_{\mu}=1$ and $\mathrm{A}_{\mu}=0.8$, shows a smoothing effect of the exterior-fluid viscosity on the interface. For $\mathrm{A}_{\mu}=0.8$, the finger has a less pronounced neck. The behavior resembles
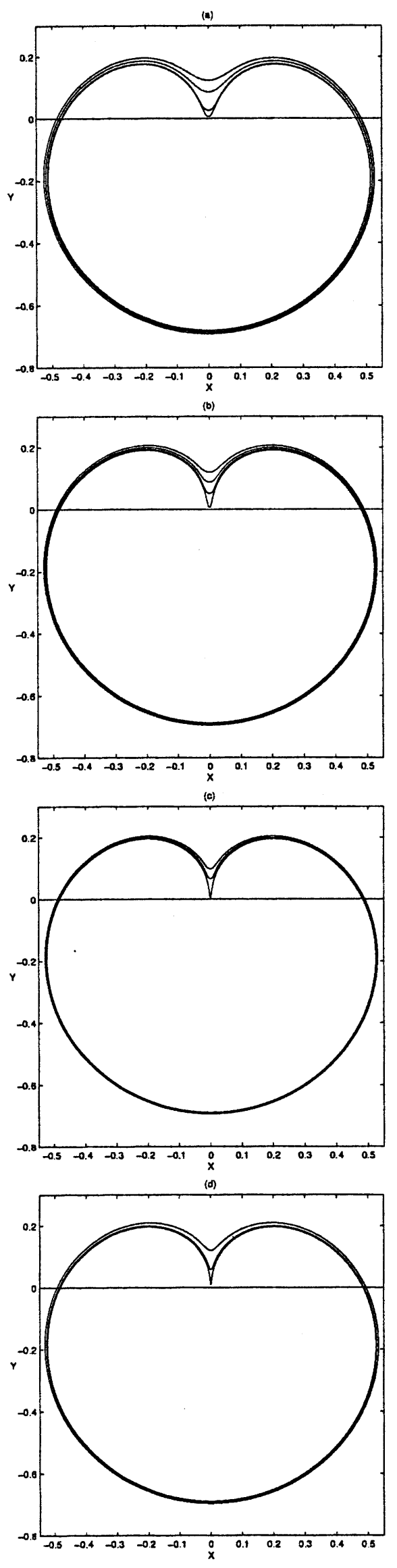

FIG. 17. Evolution of the interface for $\mathrm{A}_{\mu}=0.2$ with different surface tensions as it approaches the sink. (a) $S=1 \times 10^{-2}$, (b) $S=1 \times 10^{-3}$, (c) $S$ $=1 \times 10^{-4}$, and (d) $S=1 \times 10^{-5} . N=8192$ and $\Delta t=5 \times 10^{-6}$. 

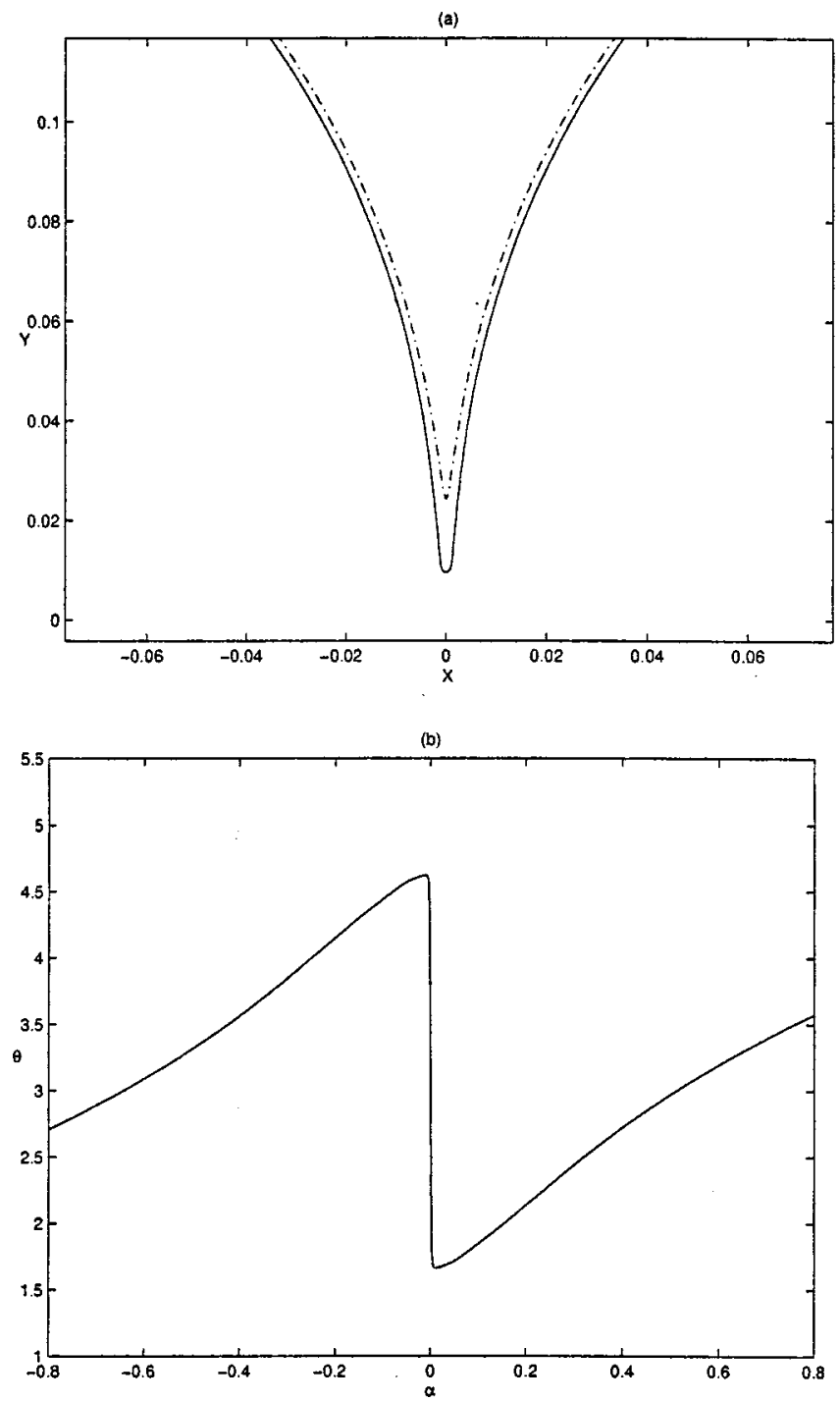

FIG. 18. Collapse of the interface for $\mathrm{A}_{\mu}=0.2$ and $S=1 \times 10^{-5}$. (a) Close-up of the finger tip at $t=0.38124$. The distance of the tip to the sink is 0.00951 . The dotted line curve corresponds to the zero-surface tension cusp at $t_{c}=0.3809$. (b) Tangent angle $\theta(\alpha, t)$ near the interface tip ( $\alpha$ $=0)$ at $t=0.38124$. This computation ended with $N=32768$ and $\Delta t=1$ $\times 10^{-7}$.

that of $\mathrm{A}_{\mu}=1$ with much larger surface tension, except that the finger is thinner for $\mathrm{A}_{\mu}=0.8$. However, the side indentations of the finger neck do develop for smaller surface tension as Fig. 15 demonstrates. The interface motion shown in this figure corresponds to $S=1 \times 10^{-5}$. We use $N=32768$ and $\Delta t=1 \times 10^{-7}$ to resolve the last stage of the flow. Note that the wedge angle is smaller than that corresponding to $\mathrm{A}_{\mu}=1$. The formation of a corner at the finger tip can be clearly appreciated in Fig. 16 which is a plot of $\theta$ around the finger at $t=0.31222$. At this time, the distance of the finger tip to the sink is $9 \times 10^{-3}$. In addition to the discontinuity at $\alpha=0$, we observe two abrupt changes in $\theta$ corresponding to the finger neck. This is analogous to the almost discontinuous transition in $\theta$ observed for $\mathrm{A}_{\mu}=1$ and $S=5 \times 10^{-6}$ in Fig. 10(b) and which we believe will lead to the formation of pair of curvature singularities at the neck in the limit as $S$ $\rightarrow 0$.

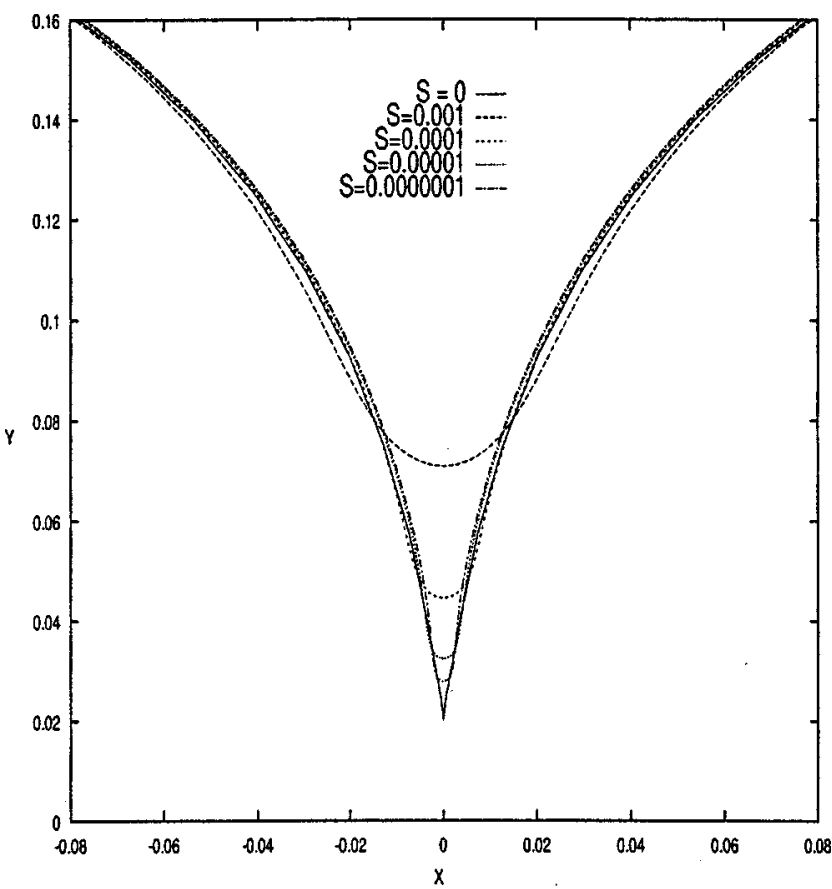

FIG. 19. Limiting behavior of the interface before $t_{c}=0.3809$ for $\mathrm{A}_{\mu}$ $=0.2$. This figure shows a close-up picture of the interface around $x=0$ for a set of surface tension values, decreasing from top to bottom, and plotted at time $t=0.3810$. The zero-surface-tension solution is also shown. $N=4096$ and $\Delta t=1 \times 10^{-5}$

\section{Surface tension and small viscosity ratio}

The Hele-Shaw flow for small Atwood ratios differs significantly from the $\mathrm{A}_{\mu}=1$ flow. Figure 17 shows the evolution of the interface for $\mathrm{A}_{\mu}=0.2$ as it approaches the sink for several values of surface tension. The interface develops a very thin finger whose width decreases with surface tension. Unlike the larger $\mathrm{A}_{\mu}$ flows, for $\mathrm{A}_{\mu}=0.2$ the zero-surfacetension cusp occurs already very close to the sink so that small surface tension acts very briefly past $t_{c}$. In the short time interval from $t_{c}$ to the time where the interface touches the sink, the sink flow is dominant and the interface profile is similar to that with zero surface tension. The effect of small surface tension is to round slightly the tip of the thin finger. This is illustrated clearly in Fig. 18(a) where a close-up picture of the finger, for $\mathrm{A}_{\mu}=0.2$ and $S=1 \times 10^{-5}$, is shown as the interface is about to collapse at $t=0.38124$. We use $N$ $=32768$ and $\Delta t=1 \times 10^{-7}$ to resolve the interface motion up to this time. The zero surface tension $\mathrm{A}_{\mu}=0.2$ cusp is also shown. Figure 18(b) is a plot of the tangent angle $\theta(\alpha, t)$ around the finger tip. This plot suggests once more the formation of a corner singularity at the finger tip as it touches the sink. Note also that there are no signs of neck formation for this case, as reflected in $\theta$.

Finally, we study the asymptotic behavior of the thin fingers for $\mathrm{A}_{\mu}=0.2$ in the limit as surface tension tends to zero. Figure 19 provides a close-up of the fingers at $t$ $=0.3800$, just before $t_{c}$. As expected, the fingers converge to the zero-surface-tension solution. To study the interface asymptotic behavior past $t_{c}$ we compare the fingers, for a decreasing set of surface tension values, when they reach a given fixed level close to the sink. This comparison is given 


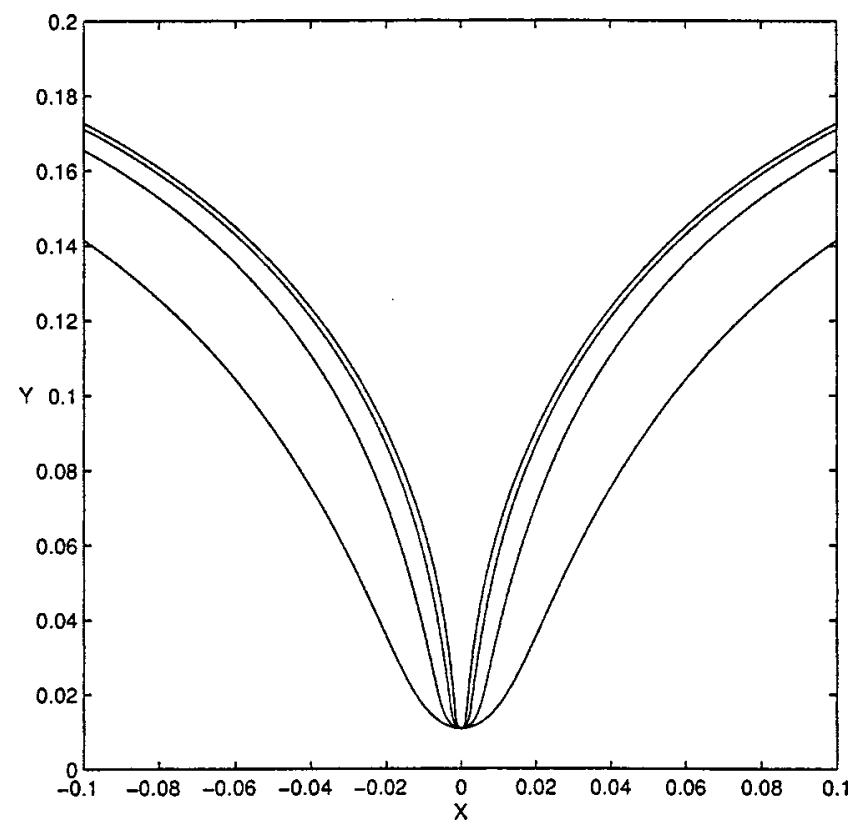

FIG. 20. Comparison of the interface finger for a sequence of surface tensions with $\mathrm{A}_{\mu}=0.2$. From the outer curve inwards, the fingers correspond to the surface tension values $S=1 \times 10^{-2}, 1 \times 10^{-3}, 1 \times 10^{-4}$, and $1 \times 10^{-5}$. Each interface is plotted when the tip of the finger reaches the fixed level $y=0.01$ at $x=0 . N=8192$ and $\Delta t=1 \times 10^{-6}$ for $S=1 \times 10^{-2}$ and $S=1$ $\times 10^{-3} . N=16384$ and $\Delta t=2 \times 10^{-7}$ for $S=1 \times 10^{-4}$ and $S=1 \times 10^{-5}$.

in Fig. 20 for surface tension ranging from $S=1 \times 10^{-2}$ to $S=1 \times 10^{-5}$. The interfaces correspond to different times past $t_{c}$. An asymptotic shape of the fingers is apparent. Table III shows the converging trend of the finger widths as surface tension is decreased. Note that the asymptotic wedge angle for $\mathrm{A}_{\mu}=0.2$ is smaller than the angles observed for $\mathrm{A}_{\mu}=0.8$ and $\mathrm{A}_{\mu}=1$. The asymptotic angle decreases with $\mathrm{A}_{\mu}$, apparently towards the zero angle of the $\mathrm{A}_{\mu}=0$ cusp.

\section{An analysis of numerical errors}

As described in the beginning of this section, our computations are performed so that all the Fourier modes of the tangent angle $\theta(\alpha, t)$ are well resolved at all times. To achieve this we successively double the number of points $\mathrm{N}$ as soon as the magnitude of the highest frequency mode of $\theta(\alpha, t)$ is greater than the filter level. The time-step size $\Delta t$ is selected so that decreasing it further would not produce any appreciable difference within plotting resolution in the curvature of the interface.

TABLE III. Change in the finger widths as surface tension is decreased for $\mathrm{A}_{\mu}=0.2$. The first column shows the height level at which the fingers are compared. Columns 2-4 give the difference $\Delta(S, S / 10)$ between the width of the finger corresponding to a surface tension $S$ and that corresponding to $S / 10$.

\begin{tabular}{cccc}
\hline \hline$y$ & $\Delta\left(10^{-2}, 10^{-3}\right)$ & $\Delta\left(10^{-3}, 10^{-4}\right)$ & $\Delta\left(10^{-4}, 10^{-5}\right)$ \\
\hline 0.04 & 0.01148 & 0.00445 & 0.00174 \\
0.03 & 0.00914 & 0.00377 & 0.00148 \\
0.02 & 0.006138 & 0.002874 & 0.001218 \\
\hline \hline
\end{tabular}

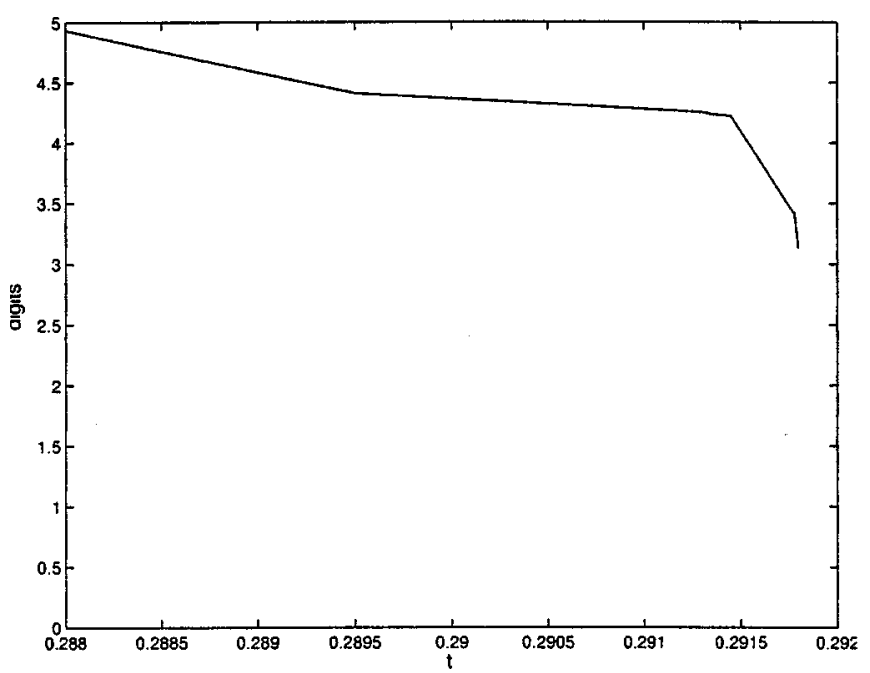

FIG. 21. The digits of accuracy in $\theta$ versus time for $S=5 \times 10^{-5}$ and $\mathrm{A}_{\mu}$ $=1$ as reflected by the maximum difference between two computations with different resolutions. One computation starts with $N=1024$ and $\Delta t=1$ $\times 10^{-4}$ and ends with $N=16384$ and $\Delta t=2 \times 10^{-7}$. The other computation uses twice the number of points and half the time step, i.e, it starts with $N$ $=2048$ and $\Delta t=5 \times 10^{-5}$, and ends with 32768 points and $\Delta t=1$ $\times 10^{-7}$.

We now present a resolution study for the long-time computation that is most difficult to resolve. This is the case corresponding to $\mathrm{A}_{\mu}=1$ and $S=5 \times 10^{-5}$ for the $10 \%$ offset initial data. In lack of an exact solution, we compare two different resolutions computed as follows. One computation starts with $N=1024$ and $\Delta t=1 \times 10^{-4}$ and ends with $N$ $=16384$ and $\Delta t=2 \times 10^{-7}$. The other computation uses twice the number of points and half the time step, i.e., it starts with $N=2048$ and $\Delta t=5 \times 10^{-5}$, and ends with 32768 points and $\Delta t=10^{-7}$. The number of digits in the maximum difference between these two resolutions for $\theta$ is presented in Fig. 21. Up to the end, the computations agree within at least 3 digits of accuracy for $\theta$. A similar resolution study for $\mathrm{A}_{\mu}=0.2$ and $S=1 \times 10^{-5}$ gives a maximum difference of $2.8 \times 10^{-4}$ at $t=0.38124$.

By monitoring closely the spectrum of the solution at all times, we verify the resolution in Fourier space and check for signs of numerical instability and noise. The spectrum of the vortex sheet strength $\gamma$ at various times for $\mathrm{A}_{\mu}=1$ and $S$ $=5 \times 10^{-5}$ flow is shown in Fig. 22. The spectra appear free of any sign of numerical instability and noise pollution is inappreciable.

Another useful check for the numerics is the conservation of the first moment for $A_{\mu}=1$ :

$$
\dot{I}=\frac{d}{d t} \iint_{\Omega(t)} z d x d y=0,
$$

where $\Omega$ is the domain of the viscous fluid and $z$ denotes the complex position of the fluid particles. This identity can be easily shown by noting that

$$
\dot{I}=\int_{\Gamma(t)} z U d s=-M \int_{\Gamma(t)} z \boldsymbol{\nabla} p \cdot \hat{\mathbf{n}} d s,
$$



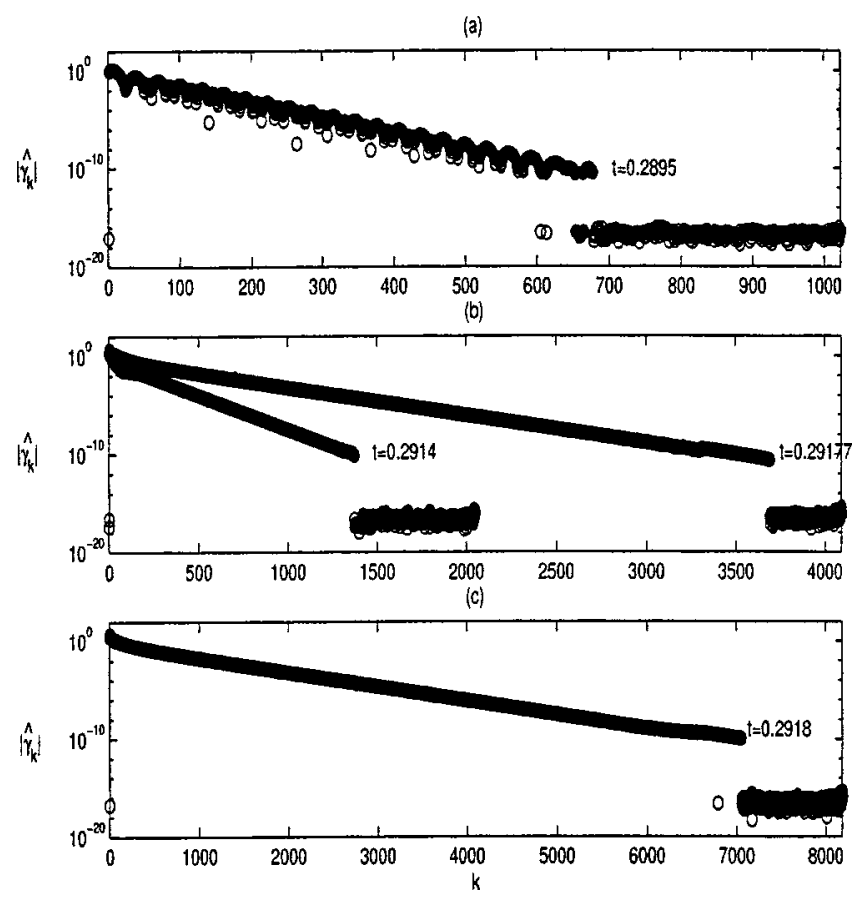

FIG. 22. The spectrum of $\gamma$ at various times for $S=5 \times 10^{-5}$ and $\mathrm{A}_{\mu}=1$.

where again $\Gamma(t)$ is the free boundary, $U$ is the normal velocity, and we have used $U=-M \nabla p \cdot \hat{\mathbf{n}}$. Since $p$ and both the real and imaginary part of $z$ are harmonic functions, a Green's identity implies that

$$
\int_{\Gamma(t)} z \boldsymbol{\nabla} p \cdot \hat{\mathbf{n}} d s=\int_{\Gamma(t)} p \boldsymbol{\nabla} z \cdot \hat{\mathbf{n}} d s .
$$

Moreover, $\left.p\right|_{\Gamma}=\tau \kappa$ for $\mathrm{A}_{\mu}=1$. Thus,

$$
\begin{aligned}
\dot{I} & =-M \int_{\Gamma(t)} z \boldsymbol{\nabla} p \cdot \hat{\mathbf{n}} d s \\
& =-M \int_{\Gamma(t)} p \boldsymbol{\nabla} z \cdot \hat{\mathbf{n}} d s \\
& =-M \tau \int_{\Gamma(t)} \kappa \nabla z \cdot \hat{\mathbf{n}} d s \\
& =i M \tau \int_{\Gamma(t)} \theta_{s} e^{i \theta} d s=i M \tau \int_{0}^{2 \pi} e^{i \theta} d \theta=0,
\end{aligned}
$$

where we have used $\kappa=\theta_{s}$. Throughout all the computations for $A_{\mu}=1,|\dot{I}|$ remains of order $10^{-12}$ or smaller.

\section{Effect of offset shift}

We consider now the effect of a shift in the position of the center of the initially circular blob. In Fig. 23, we compare a $20 \%$ offset sink [the blob center is $(0,-0.2)$ initially] with a $10 \%$ offset sink [the blob center is $(0,-0.2)$ initially], with surface tension $S=0.01$ and $\mathrm{A}_{\mu}=1.0$. Although the viscous finger forms much earlier for the $20 \%$ offset case, the width and shape of the finger are unaffected by the offset shift. We now consider a similar comparison for $S=5$ $\times 10^{-5}$ and $\mathrm{A}_{\mu}=1.0$. This is shown in Fig. 24. We find that
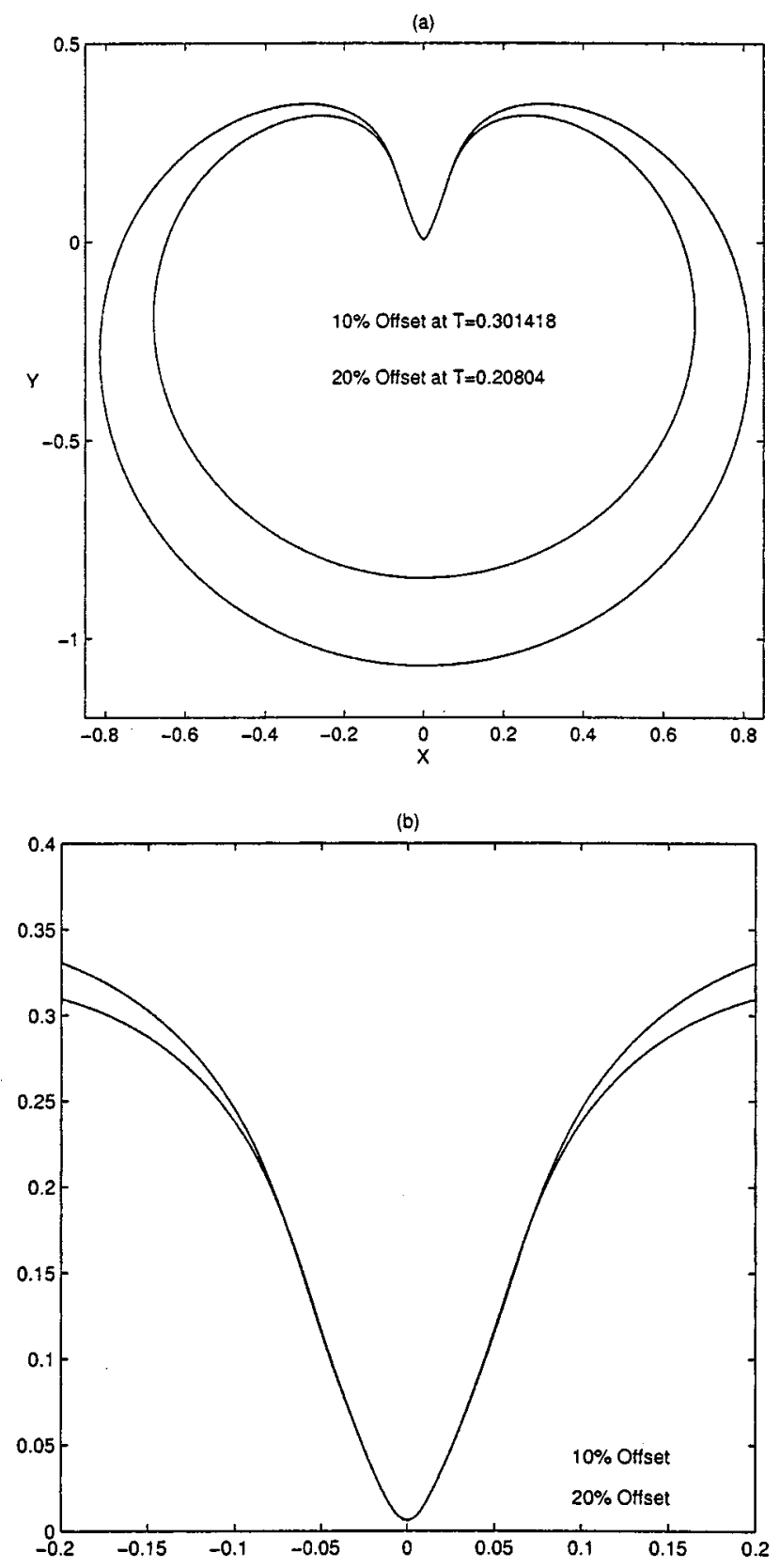

FIG. 23. Comparison of finger widths for $10 \%$ and $20 \%$ offset sinks, with $\mathrm{S}=0.01$ and $\mathrm{A}_{\mu}=1.0$. The interface for the $10 \%$ offset initial data is plotted at $t=0.301418$ and that for the $20 \%$ offset case is plotted at $t=0.20804$. (a) The two interfaces and (b) a close-up of the fingers. $N=8192$ and $\Delta t=1$ $\times 10^{-6}$ for the last stage of the motion.

the finger tips match very well and, thus, the corresponding solutions break down with the same corner angle. The overall development of the two fingers is qualitatively the same. In particular, similar side indentations at the neck are formed and the fingers slightly bulge before becoming a wedge.

\section{FURTHER DISCUSSION AND CONCLUSIONS}

Surface tension regularizes the cusped Hele-Shaw flows with suction. In the presence of small surface tension, and when the viscosity of the surrounding fluid is neglected, a long finger develops after the zero-surface-tension singularity time. This finger evolves rapidly into a wedge as it ap- 


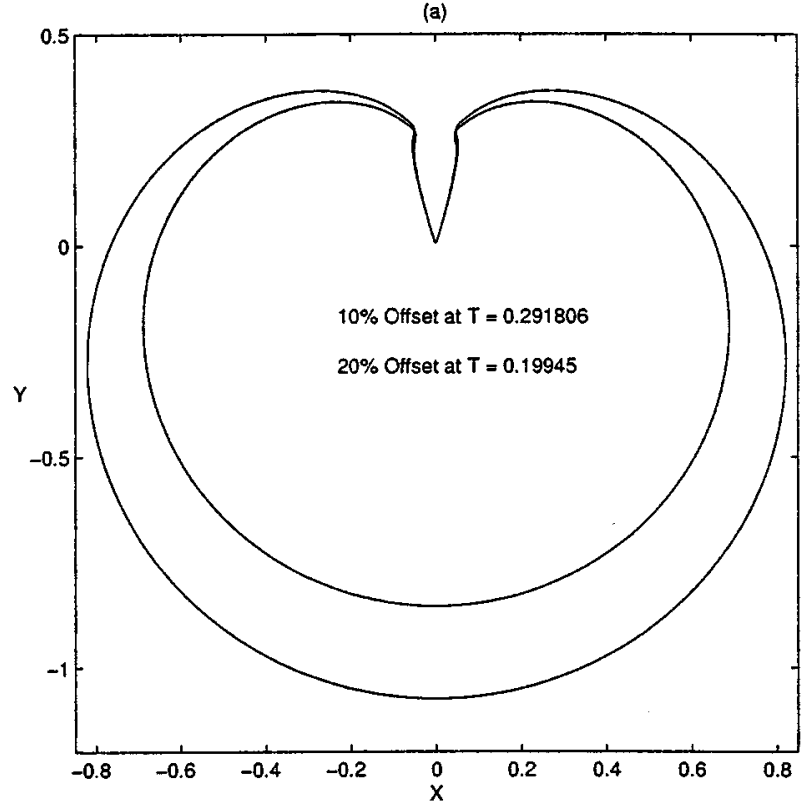

(b)

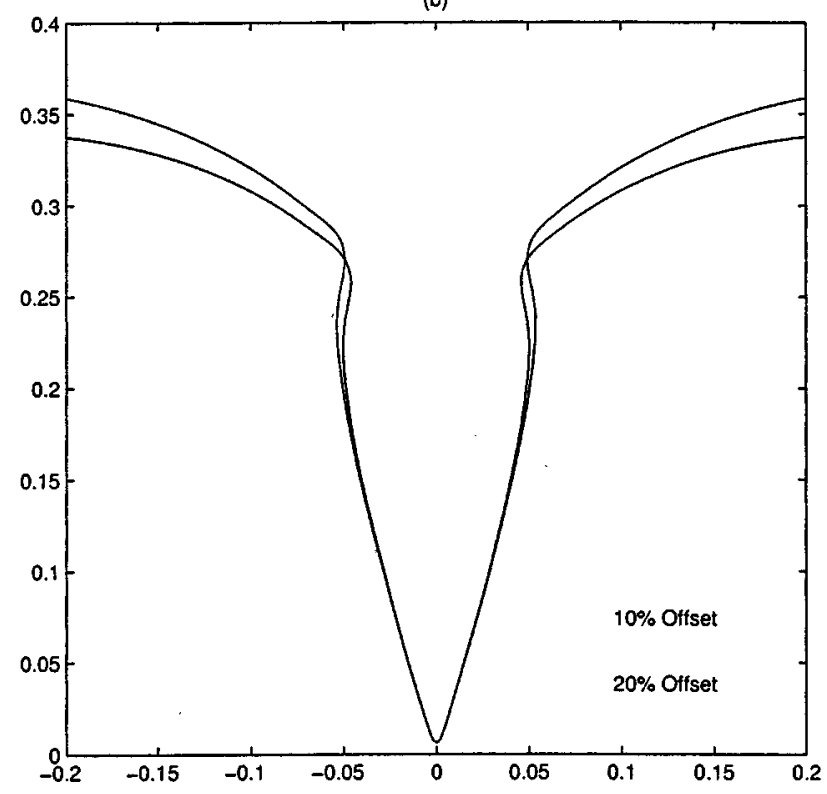

FIG. 24. Comparison of finger widths for $10 \%$ and $20 \%$ offset sinks with $S=5 \times 10^{-5}$. The interface for the $10 \%$ offset initial data is at $t$ $=0.291806$ and that for the $20 \%$ offset case is plotted at $t=0.19945 . N$ $=16384$ and $\Delta t=2 \times 10^{-7}$ for the last stage of the motion.

proaches the sink. The Hele-Shaw solutions for $\mathrm{A}_{\mu}=1$ appear to generically break down by forming a corner at the wedge tip when the interface touches the sink.

As surface tension is decreased systematically, new features of the Hele-Shaw flow are discovered. The angle at the tip of the wedge converges to a selected asymptotic value in the limit as $S \rightarrow 0$. Moreover, for sufficiently small values of surface tension, side indentations forming a neck develop at the top of the finger. As opposed to the predicted behavior of the crack model of Howison et al., ${ }^{2}$ the developing finger for $\mathrm{A}_{\mu}=1$ bulges after the zero-surface-tension cusp time $t_{c}$. The formation of the neck and the bulging of the finger are intriguing phenomena that could be linked to the influence of the zero-surface-tension singularity for very small values of surface tension.

It could also be argued that this somewhat singular behavior is caused by the impact of a curvature-induced complex singularity. This so-called daughter singularity, whose concept was introduced by Tanveer, ${ }^{22}$ is generated by a zero in the derivative of the conformal map that describes the Hele-Shaw flow. The daughter singularity is spawn by the zero through the surface tension term, in the complex plane outside the physical domain. The zero and the daughter singularity travel with different speeds towards the physical domain. Depending on the initial data, the daughter singularity may or may not impact the physical domain before the zero does. For the particular initial data that we use here, the daughter singularity would hit the physical domain well after the zero, i.e., much later than $t_{c}$. The daughter singularity impact time can be estimated by solving an ordinary differential equation. ${ }^{22,23}$ Since the neck formation and the bulging of the finger are observed around $t_{c}$, we rule out the effects of the daughter singularity for the particular flow we consider here.

The numerical evidence presented here also suggests that the limiting solution as $S \rightarrow 0$ is singular at the finger neck, for any fixed time past $t_{c}$ but before the asymptotic time at which the wedge tip touches the sink. Unfortunately, well-resolved computations are difficult to achieve due to the rapid growth of the round-off level noise for very small surface tension and to the singular interface behavior. Although further numerical study is required to compute effectively the limit, we believe that corners will develop at the finger neck in the limit as $S \rightarrow 0$. The vanishing surface tension solution selects a continuation solution past $t_{c}$ in which the zero-surface-tension cusp is split into a pair of corner singularities.

The viscosity of the exterior fluid alone does not prevent the formation of cusps. It only delays them. In the absence of surface tension, we observe generic $\frac{3}{2}$-power cusps just as in the case of $\mathrm{A}_{\mu}=1$. However, as the viscosity ratio $\mathrm{A}_{\mu}$ is decreased, we observe that the zero-surface-tension cusps develop closer and closer to the sink. We find that even for values of $\mathrm{A}_{\mu}$ as small as 0.01 , the cusp singularity develops before the interface reaches the sink. In the presence of surface tension and for large viscosity ratios, the interface evolution is very similar to that of $\mathrm{A}_{\mu}=1$ but with larger surface tension. The viscosity of the exterior fluid has a smoothing effect on the flow and a thinner finger develops. The interface behavior is significantly different for small viscosity ratios. In this case, the zero-surface-tension cusp occurs already very close to the sink. Small surface tension only rounds slightly the tip of the cusp before this part of the interface rapidly accelerates to the sink. As a result, thin cusp-like fingers develop for small viscosity ratios. These fingers appear to converge also to an asymptotic shape as surface tension is reduced. It is noted that the angle of the asymptotic wedges decreases with $\mathrm{A}_{\mu}$. We believe that this angle will collapse to zero (a cusp) in the limit as $\mathrm{A}_{\mu} \rightarrow 0$. 


\section{ACKNOWLEDGMENTS}

We would like to thank Professor Sam Howison and Professor John Ockendon for suggesting this problem to us and for their valuable comments. We also thank Dr. Mark Kunka and Professor Saleh Tanveer for a number of helpful discussions. Finally, we would like to thank Professor Stephen Cowley and Professor John Hinch for many constructive comments and suggestions. T.Y.H. acknowledges support from National Science Foundation Grant No. DMS9704976 and Office of Naval Research Grant No. N0001496-1-0438

${ }^{1}$ L. Paterson, "Radial fingering in a Hele-Shaw cell," J. Fluid Mech. 113, 513 (1981).

${ }^{2}$ S. Howison, A. Lacey, and J. Ockendon, "Hele-Shaw free-boundary problems with suction," Q. J. Mech. Appl. Math. 41, 183 (1988).

${ }^{3}$ S. Richardson, "Hele-Shaw flows with a free boundary produced by the injection of fluid into a narrow channel," J. Fluid Mech. 56, 609 (1972).

${ }^{4}$ J. Duchon and R. Robert, "Évolution d'une interface par capillarité et diffusion de volume I. Existence locale en temps," Ann. Inst. Henri Poincaré 1, 361 (1984).

${ }^{5}$ P. Constantin and M. Pugh, "Global solutions for small data to the HeleShaw problem,'” Nonlinearity 6, 393 (1993).

${ }^{6} \mathrm{~F}$. Tian, "On the breakdown of Hele-Shaw solutions with non-zero surface tension," J. Nonlinear Sci. 5, 479 (1995).

${ }^{7}$ E. DiBenedetto and A. Friedman, "The ill-posed Hele-Shaw model and the Stefan problem for supercooled water," Trans. Am. Math. Soc. 282, 183 (1984).

${ }^{8}$ S. Howison, J. Ockendon, and A. Lacey, "Singularity development in mMoving-boundary problems," Q. J. Mech. Appl. Math. 38, 343 (1985).
${ }^{9}$ M. Muskat, The Flow of Homogeneous Fluids Through Porous Media (McGraw-Hill, New York, 1937).

${ }^{10} \mathrm{~T}$. Hou, J. Lowengrub, and M. Shelley, "Removing the stiffness from interfacial flows with surface tension," J. Comput. Phys. 114, 312 (1994).

${ }^{11}$ E. Kelly and E. Hinch, "Numerical simulations of sink flow in the HeleShaw cell with small surface tension," Eur. J. Appl. Math. 8, 533 (1997).

${ }^{12}$ Q. Nie and F. R. Tian, "Singularities in Hele-Shaw flows," SIAM (Soc. Ind. Appl. Math.) J. Appl. Math. 58, 34 (1998).

${ }^{13}$ A. J. DeGregoria and L. W. Schwartz, "A boundary-integral method for two-phase displacement in Hele-Shaw cells," J. Fluid Mech. 164, 383 (1986).

${ }^{14}$ E. Meiburg and G. M. Homsy, "Nonlinear unstable viscous fingers in Hele-Shaw flows. 2. Numerical simulation," Phys. Fluids 31, 429 (1988).

${ }^{15}$ D. Bensimon, "Stability of viscous fingering," Phys. Rev. A 33, 1302 (1986)

${ }^{16}$ W. Dai and M. Shelley, "A numerical study of the effect of surface tension and noise on an expanding Hele-Shaw bubble,' Phys. Fluids A 5, 2131 (1993).

${ }^{17}$ G. Tryggvason and H. Aref, "Numerical experiments in Hele-Shaw flows with a sharp interface," J. Fluid Mech. 136, 1 (1983).

${ }^{18}$ A. Sidi and M. Israeli, "Quadrature methods for periodic singular and weakly singular Fredholm integral equations," J. Sci. Comput. 3, 201 (1988).

${ }^{19}$ U. M. Ascher, S. J. Ruuth, and B. Wetton, "Implicit-explicit methods for time-dependent partial differential equations," SIAM (Soc. Ind. Appl. Math.) J. Numer. Anal. 32, 797 (1995).

${ }^{20}$ G. Baker, D. Meiron, and S. Orszag, "Generalized vortex methods for free-surface flow problems," J. Fluid Mech. 123, 477 (1982).

${ }^{21} \mathrm{R}$. Krasny, "A study of singularity formation in a vortex sheet by the point vortex approximation,' J. Fluid Mech. 167, 65 (1986).

${ }^{22} \mathrm{~S}$. Tanveer, "Evolution of Hele-Shaw interface for small surface tension," Philos. Trans. R. Soc. London, Ser. A 343, 155 (1993).

${ }^{23}$ M. Siegel, S. Tanveer, and W. Dai, "Singular effects of surface tension in evolving Hele-Shaw flows," J. Fluid Mech. 323, 201 (1996). 